\title{
Universal Superspace Unitary Operator and Nilpotent (Anti-)Dual-BRST Symmetries: Superfield Formalism
}

\author{
T. Bhanja, ${ }^{1}$ N. Srinivas, ${ }^{1}$ and R. P. Malik ${ }^{1,2}$ \\ ${ }^{1}$ Physics Department, Centre of Advanced Studies, Banaras Hindu University, Varanasi 221 005, India \\ ${ }^{2}$ DST Centre for Interdisciplinary Mathematical Sciences, Institute of Science, Banaras Hindu University, Varanasi 221005 , India \\ Correspondence should be addressed to T. Bhanja; tapobroto.bhanja@gmail.com
}

Received 1 June 2016; Accepted 12 October 2016

Academic Editor: Edward Sarkisyan-Grinbaum

Copyright (c) 2016 T. Bhanja et al. This is an open access article distributed under the Creative Commons Attribution License, which permits unrestricted use, distribution, and reproduction in any medium, provided the original work is properly cited. The publication of this article was funded by $\mathrm{SCOAP}^{3}$.

\begin{abstract}
We exploit the key concepts of the augmented version of superfield approach to Becchi-Rouet-Stora-Tyutin (BRST) formalism to derive the superspace (SUSP) dual unitary operator and its Hermitian conjugate and demonstrate their utility in the derivation of the nilpotent and absolutely anticommuting (anti-)dual-BRST symmetry transformations for a set of interesting models of the Abelian 1-form gauge theories. These models are the one $(0+1)$-dimensional (1D) rigid rotor and modified versions of the two $(1+1)$-dimensional (2D) Proca as well as anomalous gauge theories and 2D model of a self-dual bosonic field theory. We show the universality of the SUSP dual unitary operator and its Hermitian conjugate in the cases of all the Abelian models under consideration. These SUSP dual unitary operators, besides maintaining the explicit group structure, provide the alternatives to the dual horizontality condition (DHC) and dual gauge invariant restrictions (DGIRs) of the superfield formalism. The derivations of the dual unitary operators and corresponding (anti-)dual-BRST symmetries are completely novel results in our present investigation.
\end{abstract}

\section{Introduction}

A classical gauge theory is endowed with the local gauge symmetries which are generated by the first-class constraints in the terminology of Dirac's prescription for the classification scheme $[1,2]$. Thus, one of the decisive features of a classical gauge theory is the existence of the first-class constraints on it. The above cited classical local gauge symmetries are traded with the quantum gauge [i.e., (anti-)BRST] symmetries within the framework of Becchi-Rouet-StoraTyutin (BRST) formalism. The existence of the Curci-Ferrari (CF) condition(s) [3] is one of the key signatures of a quantum gauge theory when it is BRST quantized. The geometrical superfield approach [4-10] to BRST formalism is one of the most elegant methods which leads to the derivation of the nilpotent and absolutely anticommuting (anti-)BRST transformations for a given $D$-dimensional gauge theory. In addition, this usual superfield formalism [6-8] also leads to the deduction of the (anti-)BRST invariant CF-conditions (which are the key signature of the quantum gauge theories).
Thus, we observe that, in one stroke, the usual superfield formalism (USF) produces the CF-type condition(s) as well as the proper quantum gauge [i.e., (anti-)BRST] symmetries for a quantum gauge theory. It is, therefore, clear that the USF sheds light on various aspects of quantum gauge theories when they are discussed within the framework of BRST formalism.

The USF [4-10], however, leads to the derivation of nilpotent (anti-)BRST symmetry transformations only for the gauge field and associated (anti-)ghost fields of a given quantum gauge theory. It does not shed any light on the derivation of the (anti-)BRST symmetry transformations, associated with the matter fields, in a given interacting quantum gauge theory where there is a coupling between the gauge field and matter fields. In a set of papers (see, e.g., $[11,12])$, the above superfield formalism has been consistently extended so as to derive precisely the (anti-)BRST symmetry transformations for the gauge, matter, and (anti-)ghost fields together. Whereas the usual superfield formalism exploits the theoretical potential and power of the horizontality condition 
(HC), its extended version utilizes the theoretical strength of the $\mathrm{HC}$ as well as the gauge invariant restrictions (GIRs) together in a consistent manner. The extended version of the USF has been christened $[11,12]$ as the augmented version of superfield formalism (AVSF). One of the key observations of the applications of USF and AVSF is the fact that the group structure of the (non-)Abelian gauge theories remains somewhat hidden but the geometry of these theories becomes quite explicit as we take the help of the cohomological operators of differential geometry.

The purpose of our present investigation is to exploit the theoretical strength of AVSF to derive the superspace dual unitary operators for the $1 \mathrm{D}$ and $2 \mathrm{D}$ interesting models of the Abelian 1-form gauge theories corresponding to the (anti-)dual BRST [i.e., (anti-)co-BRST] symmetry transformations which have been shown to exist for the above models. These models are the $1 \mathrm{D}$ rigid rotor and modified versions of the 2D Proca as well as anomalous gauge theories and 2D self-dual bosonic field theory. In fact, these models have been shown to provide the physical examples of Hodge theory within the framework of BRST formalism where the (anti-)BRST as well as (anti-)co-BRST symmetries exist together with a unique bosonic symmetry and the ghost-scale symmetry [13-17]. The universal superspace (SUSP) unitary operators, corresponding to the nilpotent (anti-)BRST symmetry transformations, have already been shown to exist for the above models (see, e.g., [18] for details). The central theme of our present investigation is to derive the SUSP dual unitary operators (from the above universal unitary operators). The derivation of the unitary SUSP operators is important because the group structure of the theory is maintained and it remains explicit throughout the whole discussion within the framework of AVSF. The forms of these SUSP unitary operators were first suggested in an earlier work on the superfield approach to the non-Abelian 1-form gauge theory [6-8]. These expressions, however, were intuitively chosen but not derived theoretically. Moreover, the Hermitian conjugate unitary operator, corresponding to the chosen SUSP unitary operator, was derived after imposing some outside conditions on the fields and Grassmannian variables (of the SUSP unitary operator). The sanctity of the choice, made in [6-8], was theoretically proved in our earlier work [19].

In our present investigation, we have derived the dual SUSP unitary operators (i.e., SUSP dual unitary operator and its Hermitian conjugate) which provide the alternatives to the dual horizontality condition (DHC) and dual gauge invariant restrictions (DGIRs). This derivation is a completely new result because it leads to the derivation of the nilpotent and absolutely anticommuting (anti-)co-BRST symmetry transformations which have been derived earlier within the framework of superfield approach where the DHC and DGIRs have played some decisive roles (see, e.g., [20,21]). In fact, we have obtained the proper dual SUSP unitary operator (and its Hermitian conjugate) from the universal unitary operators that have been derived in our earlier works $[19,22]$ on interacting gauge theories. To be specific, we have already derived the explicit form of the SUSP unitary operator (and its Hermitian conjugate) in the 4D interacting Abelian 1-form gauge theory with Dirac and complex scalar fields [22] as well as $4 \mathrm{D}$ non-Abelian gauge theory with Dirac fields [19]. In our present investigation, we have obtained the dual SUSP unitary operator (and its Hermitian conjugate) from the duality operation on the universal SUSP unitary operators (which have already been derived in our earlier work [22] for the $4 \mathrm{D}$ interacting Abelian theory). The form of the SUSP dual unitary operator (and its Hermitian conjugate) turns out to be universal for all the Abelian 1-form gauge models under consideration which are defined on the one- and twodimensional Minkowskian flat spacetime manifold.

Our present investigation is essential on the following key considerations. First and foremost, as we have shown the universality of the SUSP unitary operator (and its Hermitian conjugate) in the context of the models under consideration for the derivations of the (anti-)BRST symmetries, similarly, we have to derive the universal SUSP dual unitary operator (and its Hermitian conjugate) for the (anti-)coBRST transformations for the sake of completeness. We have accomplished this goal in the present investigation. Second, the existence of the SUSP dual unitary operator (and its Hermitian conjugate) provides the alternatives to the DHC and DGIRs that have been invoked in the derivation of the nilpotent (anti-)co-BRST symmetry transformations within the framework of AVSF. One of the highlights of our present investigation is the observation that the SUSP dual unitary operator and its Hermitian conjugate turn out to be universal for all the Abelian models that have been considered in our present endeavor. Third, the Abelian 1-form theories (which have been considered here) are interesting because these have been shown to provide the physical examples of Hodge theory. Fourth, we have found out the (anti-)coBRST symmetry transformation for a new model which has not been considered in our earlier works on the superfield approach to BRST formalism [20, 21]. We have obtained, for the first time, the (anti-)co-BRST symmetry transformations for the modified version of the 2D anomalous gauge theory. Thus, it is a novel result in our present endeavor. Finally, our present attempt is our modest first-step towards our central goal of establishing that these SUSP dual unitary operators are universal even in the case of non-Abelian theories.

The contents of our present investigation are organized as follows. In Section 2, we briefly discuss the (anti-)dual-BRST symmetry transformations in the Lagrangian formulation for the $1 \mathrm{D}$ rigid rotor and modified versions of the $2 \mathrm{D}$ Proca as well as anomalous gauge theories and 2D self-dual bosonic field theory. We exploit the theoretical strength of the DHC and DGIRs to derive the above nilpotent symmetries within the framework of superfield formalism in Section 3. Our Section 4 deals with the derivation of the above nilpotent symmetries by using the SUSP dual unitary operators. In Section 5, we summarize our key results and point out a few future directions for further investigation.

General Notations and Convention. We adopt the notation $s_{(a) d}$ for the on-shell as well as off-shell nilpotent (anti-)dualBRST [i.e., (anti-)co-BRST] symmetry transformations for all the $1 \mathrm{D}$ and $2 \mathrm{D}$ models under consideration. In the description of $2 \mathrm{D}$ theories, we choose $2 \mathrm{D}$ flat Minkowski metric with 
the signature $(+1,-1)$ so that the dot product between two nonnull vectors $P_{\mu}$ and $Q_{\mu}$ is defined as follows: $P \cdot Q=$ $\eta_{\mu \nu} P^{\mu} Q^{\nu}=P_{0} Q_{0}-P_{i} Q_{i}$, where the Greek indices $\mu, \nu, \lambda, \ldots=$ 0,1 correspond to the $2 \mathrm{D}$ spacetime directions and the Latin indices $i, j, k, \ldots=1$ stand for the space direction only. Our choice of the Levi-Civita tensor $\varepsilon_{\mu \nu}$ is such that $\varepsilon_{01}=+1=\varepsilon^{10}$ and $\varepsilon_{\mu \nu} \varepsilon^{\mu \nu}=+2$ !, $\varepsilon_{\mu \nu} \varepsilon^{\nu \lambda}=\delta_{\mu}^{\lambda}, \varepsilon_{\mu \nu} \varepsilon^{\mu \lambda}=-\delta_{\nu}^{\lambda}$, and so forth. The notations for the scalar and superscalar fields have been chosen to be $\phi(x)$ and $\Phi(x, \theta, \bar{\theta})$ on the 2D Minkowskian spacetime manifold and $(2,2)$-dimensional supermanifold, respectively, for all the Abelian models under consideration.

\section{Preliminaries: (Anti-)Dual-BRST Symmetries}

To begin with, we discuss here the nilpotent $\left(s_{(a) d}^{2}=0\right)$ and absolutely anticommuting $\left(s_{d} s_{a d}+s_{a d} s_{d}=0\right)$ (anti-)dualBRST symmetries $s_{(a) d}$ in the Lagrangian formulation for the $1 \mathrm{D}$ rigid rotor which is described by the following first-order Lagrangian (see, e.g., [13]):

$$
\begin{aligned}
L_{B}= & \dot{r} p_{r}+\dot{\vartheta} p_{\vartheta}-\frac{p_{\vartheta}^{2}}{2 r^{2}}-\lambda(r-a)+B\left(\dot{\lambda}-p_{r}\right)+\frac{B^{2}}{2} \\
& -i \dot{\bar{C}} \dot{C}+i \bar{C} C
\end{aligned}
$$

where the pair $(\dot{r}, \dot{\vartheta})$ is the generalized velocities corresponding to the generalized polar coordinates $(r, \vartheta)$ of the rigid rotor. We have taken the unit mass $(m=1)$ while defining the pair $\left(p_{r}, p_{\vartheta}\right)$ as the conjugate momenta corresponding to the coordinates $(r, \vartheta)$. Here $\lambda(t)$ is the "gauge" variable of the theory (which is a 1 -form $\lambda^{(1)}=d t \lambda(t)$ on a $1 \mathrm{D}$ manifold) and $B(t)$ is the Nakanishi-Lautrup type auxiliary variable. The anticommuting $(C(t) \bar{C}(t)+\bar{C}(t) C(t)=0)$ fermionic $\left(C^{2}=\bar{C}^{2}=0\right)$ (anti-)ghost variables $(\bar{C}) C$ are required to maintain the unitarity in the theory. All the variables are the function of an evolution parameter $t$ and an overdot ( $\dot{r}, \dot{\vartheta}, \dot{\lambda}, \dot{C}, \dot{\bar{C}}$, etc.) corresponds to a single derivative (i.e., $\dot{r}=d r / d t, \dot{v}=d v / d t$, etc.) with respect to $t$. It can be readily checked [13] that, under the following (anti-)dualBRST symmetry transformations $\left(s_{(a) d}\right)$ :

$$
\begin{aligned}
s_{a d} \lambda & =C, \\
s_{a d} p_{r} & =\dot{C}, \\
s_{a d} \bar{C} & =-i(r-a), \\
s_{a d}\left[r, \vartheta, B, p_{\vartheta}, C\right] & =0, \\
s_{d} \lambda & =\bar{C}, \\
s_{d} p_{r} & =\dot{\bar{C}}, \\
s_{d} C & =i(r-a), \\
s_{d}\left[r, \vartheta, B, p_{\vartheta}, \bar{C}\right] & =0,
\end{aligned}
$$

Lagrangian (1) and gauge-fixing term remain invariant $\left(s_{(a) d} L_{B}=0, s_{(a) d}\left(\dot{\lambda}-p_{r}\right)=0\right)$.

We now focus our attention on the (anti-)dual-BRST symmetry transformations for the modified version of $2 \mathrm{D}$ Proca theory (with mass parameter $m$ ) which is described by the following (anti-)BRST invariant Lagrangian density (see, e.g., $[14,15]$ for details):

$$
\begin{aligned}
\mathscr{L}_{B}^{(p)}= & \frac{1}{2}(E-m \widetilde{\phi})^{2}+m E \widetilde{\phi}-\frac{1}{2} \partial_{\mu} \widetilde{\phi} \partial^{\mu} \widetilde{\phi}+\frac{m^{2}}{2} A_{\mu} A^{\mu} \\
& +\frac{1}{2} \partial_{\mu} \phi \partial^{\mu} \phi-m A_{\mu} \partial^{\mu} \phi-\frac{1}{2}(\partial \cdot A+m \phi)^{2} \\
& -i \partial_{\mu} \bar{C} \partial^{\mu} C+i m^{2} \bar{C} C,
\end{aligned}
$$

where the 1-form $A^{(1)}=d x^{\mu} A_{\mu}$ defines the 2D gauge potential $A_{\mu}$ and the corresponding curvature tensor $F_{\mu \nu}$ is defined from the 2-form $F^{(2)}=d A^{(1)}=\left[\left(d x^{\mu} \wedge\right.\right.$ $d x^{\nu} / 2$ !) $\left.F_{\mu \nu}\right]$, where $d=d x^{\mu} \partial_{\mu}$ (with $d^{2}=0$ ) is the exterior derivative. In $2 \mathrm{D}$, the curvature tensor $F_{\mu \nu}$ contains only one independent component which is nothing but the electric field $E$. The latter turns out to be a pseudoscalar in two $(1+1)$-dimensions of spacetime. In the above, we have a pair $(\phi, \widetilde{\phi})$ of fields which is constructed by a scalar Stueckelberg field $\phi$ and a pseudoscalar field $\tilde{\phi}$. The latter has been introduced in the theory on the physical as well as mathematical grounds $[14,15]$. The fermionic $\left(C^{2}=\bar{C}^{2}=\right.$ $0, C \bar{C}+\bar{C} C=0$ ) fields are the (anti-)ghost fields $(\bar{C}) C$ which are required to maintain the unitarity in the theory. It can be readily checked that the following nilpotent $\left(s_{(a) d}^{2}=0\right)$ and absolutely anticommuting $\left(s_{d} s_{a d}+s_{a d} s_{d}=0\right)$ (anti-)dualBRST symmetry transformations $\left(s_{(a) d}\right)$

$$
\begin{aligned}
s_{a d} A_{\mu} & =-\varepsilon_{\mu \nu} \partial^{\nu} C, \\
s_{a d} C & =0, \\
s_{a d} \bar{C} & =i(E-m \tilde{\phi}), \\
s_{a d} E & =\square C, \\
s_{a d}\left(\partial \cdot A+m_{\phi}\right) & =0, \\
s_{a d} \phi & =0, \\
s_{a d} \tilde{\phi} & =-m C, \\
s_{d} A & =-\varepsilon_{\mu \nu} \partial^{\nu} \bar{C}, \\
s_{d} \bar{C} & =0, \\
s_{d} C & =-i(E-m \widetilde{\phi}), \\
s_{d} E & =\square \bar{C}, \\
\left.m_{\phi}\right) & =0, \\
s_{d} \phi & =0, \\
s_{d} \tilde{\phi} & =-m \bar{C}, \\
s_{d}\left(\partial \cdot A+{ }^{2}\right. &
\end{aligned}
$$


leave the action integral invariant because the Lagrangian density transforms to the total spacetime derivatives (see, e.g., $[14,15]$ for details). It is to be noted that the total gauge-fixing term remains invariant under $s_{(a) d}\left[\right.$ i.e., $\left.s_{(a) d}(\partial \cdot A+m \phi)=0\right]$.

Another modified version of the 2D Abelian 1-form model is the bosonized version of anomalous Abelian 1-form gauge theory which is described by the following (anti-)BRST invariant Lagrangian density (see, e.g., [16] for details):

$$
\begin{aligned}
\mathscr{L}_{B}^{(a)}= & -\frac{1}{4} F^{\mu \nu} F_{\mu \nu}+\frac{1}{2} \partial_{\mu} \phi \partial^{\mu} \phi+\frac{a}{2} A_{\mu} A^{\mu} \\
& +\left(\eta^{\mu \nu}-\varepsilon^{\mu \nu}\right) \partial_{\mu} \phi A_{\nu} \\
& +\sigma\left[(a-1)(\partial \cdot A)+\varepsilon^{\mu \nu} \partial_{\mu} A_{\nu}\right] \\
& +\frac{(a-1)}{2} \partial_{\mu} \sigma \partial^{\mu} \sigma+B(\partial \cdot A)+\frac{B^{2}}{2}+i \partial_{\mu} \bar{C} \partial^{\mu} C,
\end{aligned}
$$

where, as explained earlier, the 2-form curvature $F_{\mu \nu}$ has only electric field as its existing component and $a$ is the ambiguity parameter in the regularization of the fermionic determinant when the 2D chiral Schwinger model (with electric charge $e=1$ ) is bosonized in terms of the scalar field $\phi$. We have introduced an extra 2D bosonic field $\sigma(x)$ in the theory to convert the second-class constraints of the original $2 \mathrm{D}$ chiral Schwinger model into the first-class system so that we could have the "classical" gauge and "quantum" (anti-)BRST symmetries in the theory (see, e.g., [16] for details). The other symbols $(\bar{C}) C$ and $B(x)$ have already been explained earlier. The Lagrangian density (5) can be reexpressed as

$$
\begin{aligned}
\mathscr{L}_{B}^{(a)}= & \mathscr{B} E-\frac{\mathscr{B}^{2}}{2}+\frac{1}{2} \partial_{\mu} \phi \partial^{\mu} \phi+\frac{a}{2} A_{\mu} A^{\mu} \\
& +\left(\eta^{\mu \nu}-\varepsilon^{\mu \nu}\right) \partial_{\mu} \phi A_{\nu} \\
& +\sigma\left[(a-1)(\partial \cdot A)+\varepsilon^{\mu \nu} \partial_{\mu} A_{\nu}\right] \\
& +\frac{(a-1)}{2} \partial_{\mu} \sigma \partial^{\mu} \sigma+B(\partial \cdot A)+\frac{B^{2}}{2}+i \partial_{\mu} \bar{C} \partial^{\mu} C
\end{aligned}
$$

which is endowed with the following (anti-)co-BRST symmetries:

$$
\begin{aligned}
s_{a d} A_{\mu} & =-\varepsilon_{\mu \nu} \partial^{\nu} C, \\
s_{a d} C & =0, \\
s_{a d} \bar{C} & =-i \mathscr{B}, \\
s_{a d} \mathscr{B} & =0, \\
s_{a d} \phi & =-C, \\
s_{a d} E & =\square C, \\
s_{a d}(\partial \cdot A) & =0, \\
s_{a d} B & =0, \\
s_{a d} \sigma & =-\frac{C}{a-1} \cong C(1+a),
\end{aligned}
$$

$$
\begin{aligned}
s_{d} A_{\mu} & =-\varepsilon_{\mu \nu} \partial^{\nu} \bar{C}, \\
s_{d} \bar{C} & =0, \\
s_{d} C & =i \mathscr{B}, \\
s_{d} \mathscr{B} & =0, \\
s_{d} \phi & =-\bar{C}, \\
s_{d} E & =\square \bar{C}, \\
s_{d}(\partial \cdot A) & =0, \\
s_{d} B & =0, \\
s_{d} \sigma & =-\frac{\bar{C}}{(a-1)} \cong \bar{C}(1+a),
\end{aligned}
$$

where we have introduced an auxiliary field $\mathscr{B}(x)$ to linearize the kinetic term $\left(-(1 / 4) F_{\mu \nu} F^{\mu \nu}=(1 / 2) E^{2} \equiv \mathscr{B} E-(1 / 2) \mathscr{B}^{2}\right)$ of our modified $2 \mathrm{D}$ anomalous Abelian 1-form gauge theory. The symmetry invariance can be explicitly checked, by using the above transformations, where the action integral $S=$ $\int d^{2} x \mathscr{L}_{B}^{(a)}$ remains invariant because the above Lagrangian density transforms to the total spacetime derivatives (see, e.g., [16] for details).

Finally, we concentrate on a theoretically interesting system of the Abelian 1-form model of the 2D self-dual bosonic field theory which is described by the following (anti)BRST invariant Lagrangian density (see, e.g., [17] for details):

$$
\begin{aligned}
\mathscr{L}_{B}^{(s)}= & \frac{1}{2} \dot{\phi}^{2}-\frac{1}{2} \dot{v}^{2}+\dot{v}\left(v^{\prime}-\phi^{\prime}\right)+\lambda\left[\dot{\phi}-\dot{v}+v^{\prime}-\phi^{\prime}\right] \\
& -\frac{1}{2}\left(\phi^{\prime}-v^{\prime}\right)^{2}-\frac{1}{2}(\dot{\lambda}-v-\phi)^{2}-\dot{i \bar{C}} \dot{C} \\
& +2 i \bar{C} C,
\end{aligned}
$$

where an overdot on fields (e.g., $\dot{v}=\partial v / \partial t, \dot{\phi}=\partial \phi / \partial t$ ) corresponds to the expression for the "generalized" velocities (where a derivative with respect to the evolution parameter $t$ is taken into account) and the prime on the fields $\left(\phi^{\prime}=\right.$ $\left.\partial \phi / \partial x, v^{\prime}=\partial v / \partial x\right)$ is the space derivative with respect to the space coordinate $x$. Here $v(x)$ field is the Wess-Zumino (WZ) field and $\phi(x)$ field is the 2D self-dual bosonic field (of our present 2D self-dual field theory). The rest of the symbols have already been explained earlier. Lagrangian density (8) is endowed with the following (anti-)dual-BRST symmetry transformations $\left(s_{(a) d}\right)$ :

$$
\begin{aligned}
& s_{a d} \lambda=C, \\
& s_{a d} \phi=\frac{\dot{C}}{2}, \\
& s_{a d} v=\frac{\dot{C}}{2}, \\
& s_{a d} C=0,
\end{aligned}
$$




$$
\begin{aligned}
s_{a d} \bar{C} & =\frac{i}{2}\left(\dot{\phi}-\dot{v}+v^{\prime}-\phi^{\prime}\right), \\
s_{a d}\left(\dot{\phi}-\dot{v}+v^{\prime}-\phi^{\prime}\right) & =0, \\
s_{d} \lambda & =\bar{C}, \\
s_{d} \phi & =\frac{\dot{\bar{C}}}{2}, \\
s_{d} v & =\frac{\dot{\bar{C}}}{2}, \\
s_{d} \bar{C} & =0, \\
s_{d} C & =-\frac{i}{2}\left(\dot{\phi}-\dot{v}+v^{\prime}-\phi^{\prime}\right), \\
s_{d}\left(\dot{\phi}-\dot{v}+v^{\prime}-\phi^{\prime}\right) & =0,
\end{aligned}
$$

because Lagrangian density (8) transforms to the total "time" derivatives as

$$
\begin{aligned}
& s_{a d} \mathscr{L}_{B}^{(s)}=\frac{\partial}{\partial t}\left[\frac{\dot{C}}{2}\left(\dot{\phi}-\dot{v}+v^{\prime}-\phi^{\prime}\right)\right], \\
& s_{d} \mathscr{L}_{B}^{(s)}=\frac{\partial}{\partial t}\left[\frac{\dot{\bar{C}}}{2}\left(\dot{\phi}-\dot{v}+v^{\prime}-\phi^{\prime}\right)\right] .
\end{aligned}
$$

Thus, the action integral $S=\int d^{2} x \mathscr{L}_{B}^{(s)}$ remains invariant under $s_{(a) d}$ for the physical fields that vanish off at $t= \pm \infty$.

The decisive features of the (anti-)dual-BRST [i.e., (anti)co-BRST] symmetry transformations are the observations that (i) they are nilpotent of order two (i.e., $s_{(a) d}^{2}=0$ ) which demonstrates their fermionic nature, (ii) these nilpotent symmetries are also absolutely anticommuting $\left(s_{d} s_{a d}+\right.$ $\left.s_{a d} s_{d}=0\right)$ in nature which shows the linear independence of $s_{d}$ and $s_{a d}$, and (iii) the gauge-fixing terms, attributing their origin to the coexterior derivative (see, e.g., [1317]), remain invariant under the (anti-)dual-BRST symmetry transformations $\left(s_{(a) d}\right)$. Thus, the nomenclature of (anti)co-BRST symmetries is appropriate for these symmetries. This observation should be contrasted with the (anti-)BRST symmetries where the total kinetic term, attributing its origin to the exterior derivative, remains invariant [13-17].

\section{Nilpotent (Anti-)Co-BRST Symmetries: Superfield Approach to the Abelian 1-Form Gauge Theories}

We briefly discuss here the derivation of the (anti-)co-BRST symmetries of our 1-form gauge theories by exploiting the geometrical superfield approach to BRST formalism [412]. First of all, we focus on the derivation of the above symmetries in the context of $1 \mathrm{D}$ rigid rotor. In this connection, we note that the gauge-fixing term $\left(\dot{\lambda}-p_{r}\right)$ remains invariant under $s_{(a) d}$. Furthermore, we observe that this term has its geometrical origin in the coexterior derivative $(\delta)$ because $\delta \lambda^{(1)}=* d *(d t \lambda(t)) \equiv \dot{\lambda}(t)$, where $(*)$ is the Hodge duality operation on the $1 \mathrm{D}$ manifold. Here we have taken the 1-form as follows: $\lambda^{(1)}=d t \lambda(t)$. According to the basic tenets of AVSF, the invariance of the gauge-fixing term implies that this quantity should remain independent of the "soul" coordinates $(\theta, \bar{\theta})$ when we generalize it onto the $(1,2)$-dimensional supermanifold parameterized by the superspace coordinates $(t, \theta, \bar{\theta})$, where the pair $(\theta, \bar{\theta})$ is a set of Grassmannian variables (with $\theta^{2}=\bar{\theta}^{2}=0, \theta \bar{\theta}+\bar{\theta} \theta=$ 0 ). In older literature [23], the latter coordinates have been christened as the "soul" coordinates and $t$ has been called the body coordinate. In other words, we have the following equality:

$$
\begin{gathered}
\star \widetilde{d} \star \tilde{\lambda}^{(1)}(t, \theta, \bar{\theta})-P_{r}(t, \theta, \bar{\theta}) \\
=* d * \lambda^{(1)}(t)-p_{r}(t),
\end{gathered}
$$

where $\star$ is the Hodge duality operation on the $(1,2)$ dimensional supermanifold on which our 1D ordinary theory is generalized. The other quantities, in (11), are

$$
\begin{aligned}
d & =d t \partial_{t} \longrightarrow \\
\tilde{d} & =d t \partial_{t}+d \theta \partial_{\theta}+d \bar{\theta} \partial_{\bar{\theta}}, \\
p_{r}(t) & \longrightarrow P_{r}(t, \theta, \bar{\theta}) \\
\lambda^{(1)} & =d t \lambda(t) \longrightarrow \\
\tilde{\lambda}^{(1)} & =d t \Lambda(t, \theta, \bar{\theta})+d \theta \bar{F}(t, \theta, \bar{\theta})+d \bar{\theta} F(t, \theta, \bar{\theta}),
\end{aligned}
$$

where the supervariables $\Lambda(t, \theta, \bar{\theta}), F(t, \theta, \bar{\theta}), \bar{F}(t, \theta, \bar{\theta})$, and $P_{r}(t, \theta, \bar{\theta})$ have the following expansions along the $(\theta, \bar{\theta})$ directions of $(1,2)$-dimensional supermanifold [21]:

$$
\begin{aligned}
\Lambda(t, \theta, \bar{\theta}) & =\lambda(t)+\theta \bar{R}(t)+\bar{\theta} R(t)+i \theta \bar{\theta} S(t), \\
F(t, \theta, \bar{\theta}) & =C(t)+i \theta \bar{B}_{1}(t)+i \bar{\theta} B_{1}(t)+i \theta \bar{\theta} s(t), \\
\bar{F}(t, \theta, \bar{\theta}) & =\bar{C}(t)+i \theta \bar{B}_{2}(t)+i \bar{\theta} B_{2}(t)+i \theta \bar{\theta} \bar{s}(t), \\
P_{r}(t, \theta, \bar{\theta}) & =p_{r}(t)+\theta \bar{K}(t)+\bar{\theta} K(t)+i \theta \bar{\theta} L(t) .
\end{aligned}
$$

We note, in the above, that the secondary variables $(R, \bar{R}, s, \bar{s}$, $K, \bar{K})$ are fermionic and $\left(S, B_{1}, \bar{B}_{1}, B_{2}, \bar{B}_{2}, L\right)$ are bosonic in nature. It is elementary to verify that, in the limit $\theta=\bar{\theta}=0$, we get back our $1 \mathrm{D}$ variables $\left(\lambda, C, \bar{C}, p_{r}\right)$ that are present in Lagrangian (1). The dual horizontality condition (DHC) [cf. (11)] leads to the following [21]:

$$
\begin{aligned}
\bar{B}_{1} & =B_{2}=0, \\
s & =\bar{s}=0, \\
B_{1}+\bar{B}_{2} & =0,
\end{aligned}
$$




$$
\begin{aligned}
\bar{K} & =\dot{\bar{R}}, \\
K & =\dot{R}, \\
L & =\dot{S} .
\end{aligned}
$$

The above relationships prove that some of the secondary variables are zero and others are interconnected in a definite and precise manner. It is worthwhile to mention that the condition $B_{1}+\bar{B}_{2}=0$ is the trivial CF-type condition. This restriction is a physical condition in our theory because it is an (anti-)co-BRST invariant quantity.

We resort to the additional restrictions on the supervariables that are motivated by the basic requirements of AVSF which state that the (anti-)co-BRST invariant quantities should be independent of the "soul" coordinates. In this connection, we observe the following:

$$
\begin{aligned}
s_{(a) d}\left[\dot{r} p_{r}-i \dot{\bar{C}} \dot{C}\right] & =0, \\
s_{(a) d}[\lambda(r-a)-i \bar{C} C] & =0,
\end{aligned}
$$

which, ultimately, imply the following equalities due to DGIRs; namely,

$$
\begin{aligned}
& \dot{R}(t, \theta, \bar{\theta}) P_{r}^{(R)}(t, \theta, \bar{\theta}) \\
& \quad-i \dot{\bar{F}}^{(R)}(t, \theta, \bar{\theta}) \dot{F}^{(R)}(t, \theta, \bar{\theta})=\dot{r} p_{r}-i \dot{\bar{C}} \dot{C}, \\
& \Lambda(t, \theta, \bar{\theta})[R(t, \theta, \bar{\theta})-a] \\
& \quad-i \bar{F}^{(R)}(t, \theta, \bar{\theta}) F^{(R)}(t, \theta, \bar{\theta})=\lambda(r-a)-i \bar{C} C,
\end{aligned}
$$

where the new notations (with $R(t, \theta, \bar{\theta})=r(t)$ ) are explicitly written as

$$
\begin{aligned}
& F^{(R)}(t, \theta, \bar{\theta})=C(t)+i \bar{\theta} \mathscr{B}, \\
& \bar{F}^{(R)}(t, \theta, \bar{\theta})=\bar{C}(t)-i \theta \mathscr{B}, \\
& P^{(R)}(t, \theta, \bar{\theta})=p_{r}(t)+\theta(\dot{\bar{R}})+\bar{\theta}(\dot{R})+i \theta \bar{\theta}(\dot{S}) .
\end{aligned}
$$

In the above, we have chosen $B_{1}(t)=-\bar{B}_{2}(t)=-\mathscr{B}$ and taken the inputs from (14). Conditions (15) are now supplemented by the observations as follows: $s_{d}(\lambda \bar{C})=0$ and $s_{a d}(\lambda C)=0$. These two conditions lead to the following restrictions on the supervariables:

$$
\begin{aligned}
& \Lambda(t, \theta, \bar{\theta}) \bar{F}^{(R)}(t, \theta, \bar{\theta})=\lambda(t) \bar{C}(t), \\
& \Lambda(t, \theta, \bar{\theta}) F^{(R)}(t, \theta, \bar{\theta})=\lambda(t) C(t) .
\end{aligned}
$$

Finally, we obtain the expressions for the secondary variables in terms of the original variables of Lagrangian (1) as (see, e.g., [21] for details)

$$
\begin{aligned}
& \bar{R}=C, \\
& R=\bar{C},
\end{aligned}
$$

$$
\begin{aligned}
\mathscr{B} & =(r-a), \\
S & =(r-a) \equiv \mathscr{B} .
\end{aligned}
$$

The substitution of these values into expansions (13) and (17) leads to the following final expressions for the expansion of the supervariables (see, e.g., [21] for details):

$$
\begin{aligned}
\Lambda^{(d)}(t, \theta, \bar{\theta})= & \lambda(t)+\theta(C)+\bar{\theta}(\bar{C})+\theta \bar{\theta}[i(r-a)] \\
\equiv & \lambda(t)+\theta\left(s_{a d} \lambda\right)+\bar{\theta}\left(s_{d} \lambda\right) \\
& +\theta \bar{\theta}\left(s_{d} s_{a d} \lambda\right), \\
F^{(d)}(t, \theta, \bar{\theta})= & C(t)+\theta(0)+\bar{\theta}[i(r-a)]+\theta \bar{\theta}(0) \\
\equiv & C(t)+\theta\left(s_{a d} C\right)+\bar{\theta}\left(s_{d} C\right) \\
& +\theta \bar{\theta}\left(s_{d} s_{a d} C\right), \\
\bar{F}^{(d)}(t, \theta, \bar{\theta})= & \bar{C}(t)+\theta[-i(r-a)]+\bar{\theta}(0)+\theta \bar{\theta}(0) \\
\equiv & \bar{C}(t)+\theta\left(s_{a d} \bar{C}\right)+\bar{\theta}\left(s_{d} \bar{C}\right) \\
& +\theta \bar{\theta}\left(s_{d} s_{a d} \bar{C}\right), \\
P_{r}^{(d)}(t, \theta, \bar{\theta})= & p_{r}(t)+\theta(\dot{C})+\bar{\theta}(\dot{\bar{C}})+\theta \bar{\theta}(i \dot{r}) \\
\equiv & p_{r}(t)+\theta\left(s_{a d} p_{r}\right)+\bar{\theta}\left(s_{d} p_{r}\right) \\
& +\theta \bar{\theta}\left(s_{d} s_{a d} p_{r}\right),
\end{aligned}
$$

where the superscript $(d)$ on the supervariables denotes the expansions that have been obtained after the application of DHC and DGIRs. A careful and close look at the above expansions demonstrates that we have already obtained the nontrivial (anti-)co-BRST symmetry transformations for the variables $\left(\lambda, C, \bar{C}, p_{r}\right)$ of the $1 \mathrm{D}$ rigid rotor. The trivial nilpotent (anti-)co-BRST symmetry transformations $s_{(a) d}\left[r, p_{\theta}, \theta\right]=0$ are self-evident. It is clear that there is a geometrical meaning of $s_{(a) d}$ in the language of translational operators $\left(\partial_{\theta}, \partial_{\bar{\theta}}\right)$ along the Grassmannian directions $(\theta, \bar{\theta})$ of the $(1,2)$-dimensional supermanifold. The nilpotency $\left(\partial_{\theta}^{2}=\right.$ $\left.\partial_{\bar{\theta}}^{2}=0\right)$ and absolute anticommutativity $\left(\partial_{\theta} \partial_{\bar{\theta}}+\partial_{\bar{\theta}} \partial_{\theta}\right)$ of these generators provide the geometrical meaning to the nilpotency $\left(s_{(a) d}^{2}=0\right)$ and absolute anticommutativity $\left(s_{d} s_{a d}+s_{a d} s_{d}=0\right)$ of the (anti-)co-BRST symmetries.

We now focus on the derivation of the (anti-)co-BRST symmetry transformations $\left(s_{(a) d}\right)$ in the context of the modified versions of the 2D Proca and anomalous Abelian 1form gauge theories within the framework of AVSF. In this connection, first of all, we observe that the gauge-fixing term $(\partial \cdot A \pm m \phi)$ remains invariant [i.e., $s_{(a) d}(\partial \cdot A \pm m \phi)=0$ ] under $s_{(a) d}$ (because, separately and independently, we have $\left.s_{(a) d}(\partial \cdot A)=0, s_{(a) d} \phi=0\right)$. We note that $(\partial \cdot A)$ has its origin in the coexterior derivative $(\delta)$ because $\delta A^{(1)}=$ - $* d *\left(d x^{\mu} A_{\mu}\right)=(\partial \cdot A)$. Thus, we have to generalize 
this relationship onto the $(2,2)$-dimensional supermanifold parametrized by the superspace coordinates $\left(x^{\mu}, \theta, \bar{\theta}\right)$. Thus, according to the basic tenets of AVSF, we have the following equality (see, e.g., [15] for details):

$$
\begin{aligned}
\star \widetilde{d} \star \widetilde{A}^{(1)} & =* d * A^{(1)}, \\
\phi(x) & \longrightarrow \Phi(x, \theta, \bar{\theta})=\phi(x),
\end{aligned}
$$

where $\star$ is the Hodge duality operation on the $(2,2)$ dimensional supermanifold and other relevant symbols have already been explained earlier. In our earlier works [15], the 1.h.s. of relation (21) has been already computed clearly by taking the help of the Hodge duality operation $\star$ defined on the $(2,2)$-dimensional supermanifold [20].

At this stage, we would like to clarify some of the new symbols used in (21). We have the generalization of the ordinary exterior derivative $d=d x^{\mu} \partial_{\mu}$ and Abelian 1-form $A^{(1)}=d x^{\mu} A_{\mu}$ onto the $(2,2)$-dimensional supermanifold as

$$
\begin{aligned}
d= & d x^{\mu} \partial_{\mu} \longrightarrow \\
\tilde{d}= & d x^{\mu} \partial_{\mu}+d \theta \partial_{\theta}+d \bar{\theta} \partial_{\bar{\theta}}, \\
A^{(1)}= & d x^{\mu} A_{\mu} \longrightarrow \\
\widetilde{A}^{(1)}= & d x^{\mu} \mathscr{B}_{\mu}(x, \theta, \bar{\theta})+d \theta \bar{F}(x, \theta, \bar{\theta}) \\
& +d \bar{\theta} F(x, \theta, \bar{\theta}),
\end{aligned}
$$

where the superfields $\mathscr{B}_{\mu}(x, \theta, \bar{\theta}), F(x, \theta, \bar{\theta})$, and $\bar{F}(x, \theta, \bar{\theta})$ have the following expansions along $(\theta, \bar{\theta})$-directions of the $(2,2)$-dimensional supermanifold:

$$
\begin{aligned}
\mathscr{B}_{\mu}(x, \theta, \bar{\theta})= & A_{\mu}(x)+\theta R_{\mu}^{(1)}(x)+\bar{\theta} R_{\mu}^{(2)}(x) \\
& +i \theta \bar{\theta} S_{\mu}(x), \\
F(x, \theta, \bar{\theta})= & C(x)+i \theta B_{1}(x)+i \bar{\theta} B_{2}(x)+i \theta \bar{\theta} s(x), \\
\bar{F}(x, \theta, \bar{\theta})= & \bar{C}(x)+i \theta B_{3}(x)+i \bar{\theta} B_{4}(x)+i \theta \bar{\theta} \bar{s}(x),
\end{aligned}
$$

where $\left(A_{\mu}(x), C(x), \bar{C}(x)\right)$ are the basic fields of the modified versions of the 2D Proca and anomalous gauge theories. The set of secondary fields $\left(R_{\mu}^{(1)}, R_{\mu}^{(2)}, s, \bar{s}\right)$ are fermionic and $\left(S_{\mu}\right.$, $B_{1}, B_{2}, B_{3}, B_{4}$ ) are bosonic in nature (because of the fermionic nature of the Grassmannian variable $(\theta, \bar{\theta}))$. The dual horizontality condition (21) leads to the following very useful relationships (see, e.g., [15] for details):

$$
\begin{aligned}
\partial \cdot R^{(1)} & =\partial \cdot R^{(2)}=\partial \cdot S=0, \\
s & =\bar{s}=0 \\
B_{1} & =B_{4}=0 \\
B_{2}+B_{3} & =0
\end{aligned}
$$

where the relation $B_{2}+B_{3}=0$ is like the CF-type condition which turns out to be a trivial relationship. We would like to state that the details of (24) have been worked out in our earlier work on the superfield approach to the modified version of $2 \mathrm{D}$ Proca theory [15]. The interesting point is that the above conditions are true in the AVSF approach to the modified version of 2D anomalous gauge theory, too.

The above relations do not lead to the exact form of $R_{\mu}^{(1)}$, $R_{\mu}^{(2)}, S_{\mu}$, and $\left(B_{2}, B_{3}\right)$. The CF-type condition $B_{2}+B_{3}=0$ allows us to choose $B_{2}=\mathscr{B}$ so that $B_{3}=-\mathscr{B}$. Now, we exploit the virtue of the AVSF to derive the exact forms of the secondary fields and observe that the following (anti-)coBRST invariant quantity

$$
s_{(a) d}\left[\varepsilon^{\mu \nu}\left(\partial_{\mu} \mathscr{B}\right) A_{\nu}-i \partial_{\mu} \bar{C} \partial^{\mu} C\right]=0
$$

permits us to demand that the superfield generalization of the above quantity on the $(2,2)$-dimensional supermanifold must be independent of the soul coordinates $(\theta, \bar{\theta})$. Thus, we have the following equality:

$$
\begin{aligned}
\varepsilon^{\mu \nu} & \left(\partial_{\mu} \mathscr{B}(x)\right) \mathscr{B}_{\nu}(x, \theta, \bar{\theta}) \\
& -i \partial_{\mu} \bar{F}^{(d)}(x, \theta, \bar{\theta}) \partial^{\mu} F^{(d)}(x, \theta, \bar{\theta}) \\
\equiv & \varepsilon^{\mu \nu}\left(\partial_{\mu} \mathscr{B}(x)\right) A_{\nu}(x)-i \partial_{\mu} \bar{C}(x) \partial^{\mu} C(x) .
\end{aligned}
$$

In the above, the expansions for the superfields $F^{(d)}(x, \theta, \bar{\theta})$ and $\bar{F}^{(d)}(x, \theta, \bar{\theta})$ are

$$
\begin{aligned}
& F^{(d)}(x, \theta, \bar{\theta})=C(x)+\bar{\theta}(-i \mathscr{B}) \equiv C(x)+\bar{\theta}\left(s_{d} C\right), \\
& \bar{F}^{(d)}(x, \theta, \bar{\theta})=\bar{C}(x)+\theta(i \mathscr{B}) \equiv \bar{C}(x)+\theta\left(s_{a d} \bar{C}\right),
\end{aligned}
$$

because it is clear, from (24), that $s=\bar{s}=0$ and $B_{1}=B_{4}=$ 0 . The substitution of the explicit expansion of $F^{(d)}(x, \theta, \bar{\theta})$, $\bar{F}^{(d)}(x, \theta, \bar{\theta})$, and $\mathscr{B}_{\mu}(x, \theta, \bar{\theta})$ into (26) leads to the following relationships when we make the coefficients of $\theta, \bar{\theta}$, and $\theta \bar{\theta}$ equal to zero; namely,

$$
\begin{gathered}
\varepsilon^{\mu \nu}\left(\partial_{\mu} \mathscr{B}(x)\right) \bar{R}_{\nu}(x)+\partial_{\mu} C(x) \partial^{\mu} \mathscr{B}(x)=0, \\
\varepsilon^{\mu \nu}\left(\partial_{\mu} \mathscr{B}(x)\right) R_{\nu}(x)+\partial_{\mu} \bar{C}(x) \partial^{\mu} \mathscr{B}(x)=0, \\
\varepsilon^{\mu \nu}\left(\partial_{\mu} \mathscr{B}(x)\right) S_{\nu}(x)+\partial_{\mu} \mathscr{B}(x) \partial^{\mu} \mathscr{B}(x)=0,
\end{gathered}
$$

leading to the final determination of the secondary fields (with the help from (24)) as

$$
\begin{gathered}
\bar{R}_{\mu}(x)=-\varepsilon_{\mu \nu} \partial^{\nu} C(x), \\
R_{\mu}(x)=-\varepsilon_{\mu \nu} \partial^{\nu} \bar{C}(x), \\
S_{\mu}(x)=-\varepsilon_{\mu \nu} \partial^{\nu} \mathscr{B}(x) .
\end{gathered}
$$


Thus, we have the following explicit expansions of the superfield:

$$
\begin{aligned}
\mathscr{B}_{\mu}^{(d)}(x, \theta, \bar{\theta})= & A_{\mu}(x)+\theta\left(-\varepsilon_{\mu \nu} \partial^{\nu} C(x)\right) \\
& +\bar{\theta}\left(-\varepsilon_{\mu \nu} \partial^{\nu} \bar{C}(x)\right) \\
& +\theta \bar{\theta}\left[-i \varepsilon_{\mu \nu} \partial^{\nu} \mathscr{B}(x)\right] \\
\equiv & A_{\mu}(x)+\theta\left(s_{a d} A_{\mu}(x)\right) \\
& +\bar{\theta}\left(s_{d} A_{\mu}(x)\right)+\theta \bar{\theta}\left(s_{d} s_{a d} A_{\mu}(x)\right) .
\end{aligned}
$$

It is very clear that we have derived the following (anti-)coBRST symmetry transformations for the fields $\left(A_{\mu}(x), C(x)\right.$, $\bar{C}(x))$ due to superfield formalism:

$$
\begin{aligned}
s_{d} A_{\mu} & =-\varepsilon_{\mu \nu} \partial^{\nu} \bar{C}, \\
s_{a d} A_{\mu} & =-\varepsilon_{\mu \nu} \partial^{\nu} C, \\
s_{d} s_{a d} A_{\mu} & =-i \varepsilon_{\mu \nu} \partial^{\nu} \mathscr{B}, \\
s_{d} C & =-i \mathscr{B}, \\
s_{a d} C & =0, \\
s_{d} s_{a d} C & =0, \\
s_{d} \bar{C} & =0, \\
s_{a d} \bar{C} & =i \mathscr{B}, \\
s_{d} s_{a d} \bar{C} & =0 .
\end{aligned}
$$

The nilpotency and absolute anticommutativity properties of the (anti-)co-BRST symmetry transformations imply that we have $s_{(a) d} \mathscr{B}=0$. Up to this point, our results are the same for the superfield description of the modified versions of 2D Proca and anomalous gauge theories because the above transformations are common to both the above theories.

To determine the (anti-)dual-BRST symmetry transformations for the $\tilde{\phi}(x)$ field of the modified version of the 2D Proca theory (cf. (3)), we observe that $s_{(a) d}\left[A_{\mu}-\right.$ $\left.(1 / m) \varepsilon_{\mu \nu} \partial^{\nu} \widetilde{\phi}\right]=0$. Thus, according to the basic requirements of AVSF, we demand that this quantity should remain independent of the "soul" coordinates; namely,

$$
\begin{gathered}
\mathscr{B}_{\mu}^{(d)}(x, \theta, \bar{\theta})-\frac{1}{m} \varepsilon_{\mu \nu} \partial^{\nu} \widetilde{\Phi}(x, \theta, \bar{\theta}) \\
=A_{\mu}(x)-\frac{1}{m} \varepsilon_{\mu \nu} \partial^{\nu} \widetilde{\phi}(x) .
\end{gathered}
$$

Now if we take the expansion of the superfield

$$
\widetilde{\Phi}(x, \theta, \bar{\theta})=\widetilde{\phi}(x)+\theta f_{4}(x)+\bar{\theta} f_{5}(x)+i \theta \bar{\theta} b_{4}(x),
$$

we obtain, from (32), the relationships $f_{4}(x)=-m C, f_{5}(x)=$ $-m \bar{C}$, and $b_{4}(x)=-m \mathscr{B}(x)$ which show the fermionic nature of $\left(f_{4}, f_{5}\right)$ and bosonic nature of $b_{4}$. Thus, the final expansion of (33), in terms of the (anti-)co-BRST symmetries $s_{(a) d}$, is

$$
\begin{aligned}
\widetilde{\Phi}^{(d)}(x, \theta, \bar{\theta})= & \widetilde{\phi}(x)+\theta(-m C)+\bar{\theta}(-m \bar{C}) \\
& +\theta \bar{\theta}(i m \mathscr{B}) \\
\equiv & \widetilde{\phi}(x)+\theta\left(s_{a d} \widetilde{\phi}\right)+\bar{\theta}\left(s_{d} \widetilde{\phi}\right) \\
& +\theta \bar{\theta}\left(s_{d} s_{a d} \widetilde{\phi}\right) .
\end{aligned}
$$

We have, therefore, derived all the nontrivial (anti-)co-BRST symmetry transformations for the fields $A_{\mu}, C, \bar{C}$, and $\widetilde{\phi}$ of the modified version of $2 \mathrm{D}$ Proca theory. The rest of the transformations are trivial (e.g., $s_{(a) d} \phi=0$ and $s_{(a) d} \mathscr{B}=0$ ) and they can be derived in a straightforward manner from the AVSF because $\phi(x) \rightarrow \Phi(x, \theta, \bar{\theta})=\phi(x)$ and $\mathscr{B}(x) \rightarrow$ $\widetilde{\mathscr{B}}(x, \theta, \bar{\theta})=\mathscr{B}(x)$. We reemphasize that transformations (31) are common to the modified versions of 2D Proca and anomalous gauge theories. As far as the latter theory is concerned, we have to derive the (anti-)co-BRST symmetry transformations for the scalar fields $\phi(x)$ and $\sigma(x)$. In this connection, we observe that the following useful quantities are (anti-)co-BRST invariant; namely, $s_{(a) d}[E+\square \phi]=0$ and $s_{(a) d}[(a-1) \sigma-\phi(x)]=0$. It is to be noted that $E=$ $-\varepsilon^{\mu \nu} \partial_{\mu} A_{\nu}=\partial_{0} A_{1}-\partial_{1} A_{0}$ in $2 \mathrm{D}$. Thus, according to the basic requirement of AVSF, we have the following equality due to the restriction on the superfield:

$$
\begin{gathered}
-\varepsilon^{\mu \nu} \partial_{\mu} \mathscr{B}_{\nu}^{(d)}(x, \theta, \bar{\theta})+\square \Phi(x, \theta, \bar{\theta}) \\
=-\varepsilon^{\mu \nu} \partial_{\mu} A_{\nu}(x)+\square \phi(x),
\end{gathered}
$$

where the expansions for $\mathscr{B}_{\mu}^{(d)}(x, \theta, \bar{\theta})$ and $\Phi(x, \theta, \bar{\theta})$ are given in (30) and (42) (see below). Substitution of these values into (35) yields the following relationships: $\bar{f}_{1}=-C, f_{1}=-\bar{C}$, and $b_{1}=-\mathscr{B}$ which imply the following expansions for the scalar superfield $\Phi^{(d)}(x, \theta, \bar{\theta})$ after the application of DGIRs; namely,

$$
\begin{aligned}
\Phi^{(d)}(x, \theta, \bar{\theta})= & \phi(x)+\theta(-C)+\bar{\theta}(-\bar{C})+\theta \bar{\theta}(-i \mathscr{B}) \\
\equiv & \phi(x)+\theta\left(s_{a d} \phi\right)+\bar{\theta}\left(s_{d} \phi\right) \\
& +\theta \bar{\theta}\left(s_{d} s_{a d} \phi\right) .
\end{aligned}
$$

We have to determine the (anti-)co-BRST transformations on the field $\sigma(x)$. In this regard, we have the following equality due to AVSF:

$$
\begin{aligned}
& (a-1) \Sigma(x, \theta, \bar{\theta})-\Phi^{(d)}(x, \theta, \bar{\theta}) \\
& =(a-1) \sigma(x)-\phi(x),
\end{aligned}
$$

where the expansion of $\Phi^{(d)}(x, \theta, \bar{\theta})$ is given in (36) and we have taken the following general expansions of $\Sigma(x, \theta, \bar{\theta})$ along the Grassmannian $(\theta, \bar{\theta})$-directions of the $(2,2)$ dimensional supermanifold; namely,

$$
\Sigma(x, \theta, \bar{\theta})=\sigma(x)+\theta \bar{P}(x)+\bar{\theta} P(x)+i \theta \bar{\theta} Q(x),
$$


where the secondary fields $(P(x), \bar{P}(x))$ are fermionic and $Q(x)$ is bosonic (due to the fermionic nature of $\theta$ and $\bar{\theta}$ ). It is straightforward to observe, from (37), that we have

$$
\begin{aligned}
P(x) & =\frac{-\bar{C}}{(a-1)}, \\
\bar{P}(x) & =\frac{-C}{(a-1)}, \\
Q & =\frac{-\mathscr{B}}{(a-1)} .
\end{aligned}
$$

The above values imply that the superexpansion (38) is

$$
\begin{aligned}
\Sigma^{(d)}(x, \theta, \bar{\theta})= & \sigma(x)+\theta\left(s_{a d} \sigma\right)+\bar{\theta}\left(s_{d} \sigma\right) \\
& +\theta \bar{\theta}\left(s_{d} s_{a d} \sigma(x)\right),
\end{aligned}
$$

where $s_{d} \sigma=-(\bar{C} /(a-1)), s_{a d} \sigma=-(C /(a-1))$, and $s_{d} s_{a d}=-(i \mathscr{B} /(a-1))$. Thus, we have derived all the nontrivial (anti-)co-BRST symmetry transformations for the modified version of $2 \mathrm{D}$ anomalous gauge theory within the framework of AVSF.

We are now in the position to discuss the superfield approach to the derivation of the (anti-)co-BRST symmetries for the 2D self-dual chiral bosonic field theory. First of all, we generalize the relevant fields of the $2 \mathrm{D}$ theory onto the $(2,2)$-dimensional superfield parametrized by the superspace coordinates $\left(x^{\mu}, \theta, \bar{\theta}\right)$ as

$$
\begin{gathered}
\phi(x) \longrightarrow \Phi(x, \theta, \bar{\theta}), \\
v(x) \longrightarrow V(x, \theta, \bar{\theta}), \\
C(x) \longrightarrow F(x, \theta, \bar{\theta}), \\
\bar{C}(x) \longrightarrow \bar{F}(x, \theta, \bar{\theta}), \\
\lambda(x) \longrightarrow \Lambda(x, \theta, \bar{\theta}),
\end{gathered}
$$

which have the following expansions along the Grassmannian directions (i.e., $(\theta, \bar{\theta})$-directions) of the $(2,2)$-dimensional supermanifold [17]:

$$
\begin{aligned}
& \Phi(x, \theta, \bar{\theta})=\phi(x)+i \theta \bar{f}_{1}(x)+i \bar{\theta} f_{1}(x)+i \theta \bar{\theta} b_{1}(x), \\
& V(x, \theta, \bar{\theta})=v(x)+i \theta \bar{f}_{2}(x)+i \bar{\theta} f_{2}(x)+i \theta \bar{\theta} b_{2}(x), \\
& F(x, \theta, \bar{\theta})=C(x)+i \theta \bar{B}_{1}(x)+i \bar{\theta} B_{1}(x)+i \theta \bar{\theta} s(x), \\
& \bar{F}(x, \theta, \bar{\theta})=\bar{C}(x)+i \theta \bar{B}_{2}(x)+i \bar{\theta} B_{2}(x)+i \theta \bar{\theta} \bar{s}(x), \\
& \Lambda(x, \theta, \bar{\theta})=\lambda(x)+\theta \bar{R}(x)+\bar{\theta} R(x)+i \theta \bar{\theta} S(x),
\end{aligned}
$$

where the set $\left(S, B_{1}, \bar{B}_{1}, B_{2}, \bar{B}_{2}, b_{1}, b_{2}\right)$ is made up of the bosonic secondary fields and the fermionic secondary fields are $\left(R, \bar{R}, s, \bar{s}, f_{1}, \bar{f}_{1}, f_{2}, \bar{f}_{2}\right)$. We obtain the basic fields $(\lambda, \phi, v, C, \bar{C})$ of the theory in the limit $\theta=\bar{\theta}=0$. We shall obtain the exact expressions for the secondary fields in terms of the basic and auxiliary fields of the theory by exploiting the physically motivated restrictions on the superfields. First of all, we take into account the appropriate generalizations of the exterior derivative and connection 1 -form onto the $(2,2)$ dimensional supermanifold, as [17]

$$
\begin{aligned}
d & \longrightarrow \\
\tilde{d} & =d t \partial_{t}+d \theta \partial_{\theta}+d \bar{\theta} \partial_{\bar{\theta}}, \\
\lambda^{(1)} & \longrightarrow \\
\tilde{\lambda}^{(1)} & =d t \Lambda(x, \theta, \bar{\theta})+d \theta \bar{F}(x, \theta, \bar{\theta})+d \bar{\theta} F(x, \theta, \bar{\theta}) .
\end{aligned}
$$

It should be noted that even though we have generalized the ordinary theory onto the $(2,2)$-dimensional supermanifold, the superexterior derivative $(\widetilde{d})$ has been defined on the $(1,2)$ dimensional supersubmanifold. This is due to the peculiarity of the gauge field in the case of $2 \mathrm{D}$ self-dual bosonic field theory where only one component of the $2 \mathrm{D}$ gauge field couples with the matter fields but the other component of the gauge field remains inert (see, e.g., [17] for details). The basic tenets of AVSF state that all the (anti-)co-BRST invariant quantities should be independent of the "soul" coordinates $(\theta, \bar{\theta})$. In this connection, we note that the following are the (anti-)co-BRST invariant quantities (see, e.g., [17]):

$$
\begin{aligned}
s_{(a) d}[\phi-v] & =0, \\
s_{(a) d}[\dot{\lambda}-\phi-v] & =0, \\
s_{(a) d}\left[\dot{\phi}-\dot{v}+v^{\prime}-\phi^{\prime}\right] & =0 .
\end{aligned}
$$

Thus, the above quantities in the square brackets, when generalized on the $(2,2)$-dimensional supermanifold, should be independent of the "soul" coordinates $(\theta, \bar{\theta})$. Plugging in the expansions from (42), we obtain the following:

$$
\begin{aligned}
& \bar{f}_{1}=\bar{f}_{2} \equiv \bar{f}, \\
& f_{1}=f_{2} \equiv f, \\
& b_{1}=b_{2} \equiv b, \\
& \dot{\bar{R}}=2 i \bar{f}, \\
& \dot{R}=2 i f, \\
& \dot{S}=2 b .
\end{aligned}
$$

We shall see that these relationships would be useful in our further discussions. For instance, we observe that the following are the invariant quantities:

$$
\begin{aligned}
& s_{(a) d}[\dot{\lambda}-2 \phi]=0, \\
& s_{(a) d}[\dot{\lambda}-2 v]=0 .
\end{aligned}
$$

In the above expressions, it is elementary to note that $\delta \lambda^{(1)}=$ $+* d * \lambda^{(1)}$ is nothing but $\dot{\lambda}$ (i.e., $\delta \lambda^{(1)}=+* d *(d t \lambda(x))=$ 
$\dot{\lambda}(x))$. We have to generalize this relationship on the $(2,2)-$ dimensional supermanifold as

$$
\begin{aligned}
\tilde{\delta} \tilde{\lambda}^{(1)}(x, \theta, \bar{\theta})-2 \Phi(x, \theta, \bar{\theta}) & =\delta \lambda^{(1)}(x)-2 \phi(x), \\
\lambda^{(1)}(x) & =d t \lambda(x),
\end{aligned}
$$

where $\widetilde{\delta}=\star \widetilde{d} \star$. Here $\star$ is the Hodge duality operation on the $(1,2)$-dimensional supersubmanifold of the general $(2,2)$ dimensional supermanifold and $\widetilde{\delta}$ is the super coexterior derivative (with $\widetilde{d}=d t \partial_{t}+d \theta \partial_{\theta}+d \bar{\theta} \partial_{\bar{\theta}}$ ). It is to be noted that the gauge field $\lambda$ is a function of $x^{\mu}(\mu=0,1)$ but the geometrical quantities $\widetilde{d}$ and $\widetilde{\delta}$ as well as $d=d t \partial_{t}$ and $\delta=* d *$ are defined in terms of $t$ only. In other words, $d$ and $\delta$ are defined on the 1D submanifold of the 2D ordinary Minkowskian spacetime manifold and $\widetilde{d}$ and $\widetilde{\delta}$ are defined on the (1,2)-dimensional supersubmanifold of the $(2,2)$ dimensional supermanifold on which our ordinary 2D theory is generalized. The 1.h.s. of (47) has been worked out in our earlier work [21]. The following relationship emerges from (47):

$$
\begin{aligned}
B_{1}+\bar{B}_{2} & =0 \Longrightarrow \\
B_{1} & =-\mathscr{B}=-\bar{B}_{2} .
\end{aligned}
$$

This condition is nothing but the analogue of the CF-type restriction which is essential as far as the proof of the absolute anticommutativity property (i.e., $s_{d} s_{a d}+s_{a d} s_{d}=0$ ) of the nilpotent (anti-)dual-BRST symmetry transformations $s_{(a) d}$ is concerned. This condition is also (anti-)dual-BRST invariant under the above symmetry transformations $s_{(a) d}$. Thus, this restriction is a physical condition on the model under consideration within the realm of BRST formalism. In fact, the whole theory is defined on the constrained hypersurface (defined by the above trivial constrained condition) that is embedded in the 2D Minkowskian spacetime manifold on which the whole of our present theory is defined.

Ultimately, we concentrate on the following (anti-)coBRST invariance:

$$
s_{(a) d}\left[\lambda\left(\dot{\phi}-\dot{v}+v^{\prime}-\phi^{\prime}\right)+2 i \bar{C} C\right]=0
$$

which imply the following restriction on the supervariables [17]:

$$
\begin{aligned}
\Lambda & {\left[\dot{\Phi}-\dot{V}+V^{\prime}-\Phi^{\prime}\right]+2 i \bar{F}^{(d)} \bar{F}^{(d)} } \\
& =\lambda\left(\dot{\phi}-\dot{v}+v^{\prime}-\phi^{\prime}\right)+2 i \bar{C} C
\end{aligned}
$$

where the expansions for $F^{(d)}$ and $\bar{F}^{(d)}$ are as follows [17]:

$$
\begin{aligned}
F^{(d)}(x, \theta, \bar{\theta}) & =C(x)+\bar{\theta}(-i \mathscr{B}(x)) \\
& \equiv C(x)+\bar{\theta}\left(s_{d} C(x)\right), \\
\bar{F}^{(d)}(x, \theta, \bar{\theta}) & =\bar{C}(x)+\theta(+i \mathscr{B}(x)) \\
& \equiv \bar{C}(x)+\theta\left(s_{a d} \bar{C}(x)\right) .
\end{aligned}
$$

Here the superscript $(d)$ denotes the superexpansions obtained after the application of DHC given in (47). Plugging in the expressions from (43) and (51), we obtain

$$
\begin{aligned}
& \bar{R}=+C, \\
& R=+\bar{C}, \\
& S=-\mathscr{B},
\end{aligned}
$$

which imply the following:

$$
\begin{aligned}
f & =-\frac{i}{2} \dot{\bar{C}}, \\
\bar{f} & =-\frac{i}{2} \dot{C}, \\
b & =-\frac{1}{2} \dot{\mathscr{B}} .
\end{aligned}
$$

At this stage, we are free to choose the auxiliary field $\mathscr{B}$ in such a manner that $s_{(a) d} \mathscr{B}=0$. The latter condition is essential because of the requirements of nilpotency and absolute anticommutativity. We choose the following in terms of the basic fields as [17]

$$
\mathscr{B}=+\frac{1}{2}\left[\dot{\phi}-\dot{v}+v^{\prime}-\phi^{\prime}\right],
$$

which serves our purpose. Finally, we have the following expansions (see, e.g., [17]):

$$
\begin{aligned}
\Lambda^{(d)}(x, \theta, \bar{\theta})= & \lambda(x)+\theta(C)+\bar{\theta}(\bar{C}) \\
& +\theta \bar{\theta}\left[-\frac{i}{2}\left(\dot{\phi}-\dot{v}+v^{\prime}-\phi^{\prime}\right)\right] \\
\equiv & \lambda(x)+\theta\left(s_{a d} \lambda\right)+\bar{\theta}\left(s_{d} \lambda\right) \\
& +\theta \bar{\theta}\left(s_{d} s_{a d} \lambda\right), \\
F^{(d)}(x, \theta, \bar{\theta})= & C(x)+\theta(0) \\
& +\bar{\theta}\left(-\frac{i}{2}\left[\dot{\phi}-\dot{v}+v^{\prime}-\phi^{\prime}\right]\right)+\theta \bar{\theta}(0) \\
\equiv & C(x)+\theta\left(s_{a d} C\right)+\bar{\theta}\left(s_{d} C\right) \\
& +\theta \bar{\theta}\left(s_{d} s_{a d} C\right), \\
& +\theta \bar{\theta}\left(-\frac{i}{4} \frac{\partial}{\partial t}\left[\dot{\phi}-\dot{v}+v^{\prime}-\phi^{\prime}\right]\right) \\
\Phi^{(d)}(x, \theta, \bar{\theta})= & \phi(x)+\theta\left(+\frac{\dot{C}}{2}\right)+\bar{\theta}\left(+\frac{\bar{C}}{2}\right) \\
\bar{F}^{(d)}(x, \theta, \bar{\theta})= & +\theta \bar{\theta}\left(s_{d} s_{a d} \bar{C}\right), \theta\left(\frac{i}{2}\left[\dot{\phi}-\dot{v}+v^{\prime}-\phi^{\prime}\right]\right) \\
& +\bar{\theta}(0)+\theta \bar{\theta}(0) \\
\equiv & \bar{C}(x)+\theta\left(s_{a d} \bar{C}\right)+\bar{\theta}\left(s_{d} \bar{C}\right) \\
& \\
& \\
& \\
&
\end{aligned}
$$




$$
\begin{aligned}
\equiv & \phi(x)+\theta\left(s_{a d} \phi\right)+\bar{\theta}\left(s_{d} \phi\right) \\
& +\theta \bar{\theta}\left(s_{d} s_{a d} \phi\right), \\
V^{(d)}(x, \theta, \bar{\theta})= & v(x)+\theta\left(+\frac{\dot{C}}{2}\right)+\bar{\theta}\left(+\frac{\dot{\bar{C}}}{2}\right) \\
& +\theta \bar{\theta}\left(-\frac{i}{4} \frac{\partial}{\partial t}\left[\dot{\phi}-\dot{v}+v^{\prime}-\phi^{\prime}\right]\right) \\
\equiv & v(x)+\theta\left(s_{a d} v\right)+\bar{\theta}\left(s_{d} v\right) \\
& +\theta \bar{\theta}\left(s_{d} s_{a d} v\right),
\end{aligned}
$$

where the superscript $(d)$ denotes the expansion of the superfields after the imposition of the DHC and DGIRs within the framework of AVSF. Thus, we note that we have derived all the (anti-)co-BRST symmetry transformations listed in (9). The nilpotency and absolute anticommutativity of $s_{(a) d}$ imply that $s_{(a) d}\left[\dot{\phi}-\dot{v}+v^{\prime}-\phi^{\prime}\right]=0$.

\section{SUSP Dual Unitary Operator: Universal Aspects}

The precise expressions for the SUSP unitary operator and its Hermitian conjugate have been explicitly derived in our earlier work [22] on the 4D interacting Abelian 1-form gauge theory with Dirac and complex scalar fields where we have provided the alternatives to the $\mathrm{HC}$ and GIRs in the context of the derivation of the (anti-)BRST symmetries of this theory. These forms are expressed, in terms of the familiar symbols, as follows:

$$
\begin{aligned}
U(x, \theta, \bar{\theta}) & =1+\theta(-i \bar{C})+\bar{\theta}(-i C)+\theta \bar{\theta}(B-C \bar{C}), \\
U^{\dagger}(x, \theta, \bar{\theta}) & =1+\theta(i \bar{C})+\bar{\theta}(i C)+\theta \bar{\theta}(-B-C \bar{C}),
\end{aligned}
$$

which satisfy $U U^{\dagger}=U^{\dagger} U=1$. It is important to point out that the above explicit expressions have been derived by exploiting the theoretical strength behind the concept of covariant derivatives. Expressions (56) can be also written in the exponential forms as

$$
\begin{aligned}
U(x, \theta, \bar{\theta}) & =\exp [\theta(-i \bar{C})+\bar{\theta}(-i C)+\theta \bar{\theta} B], \\
U^{\dagger}(x, \theta, \bar{\theta}) & =\exp [\theta(i \bar{C})+\bar{\theta}(i C)-\theta \bar{\theta} B],
\end{aligned}
$$

which very clearly demonstrate the validity of unitary condition: $U U^{\dagger}=U^{\dagger} U=1$. The basic idea behind the covariant derivative also leads to the transformation property of the 1form $A^{(1)}=d x^{\mu} A_{\mu}$ gauge connection under the (anti-)BRST symmetry transformations, in the language of SUSP unitary operator and its Hermitian conjugate, as $[18,22]$

$$
\begin{aligned}
\widetilde{A}_{(h)}^{(1)}= & U(x, \theta, \bar{\theta}) A^{(1)}(x) U^{\dagger}(x, \theta, \bar{\theta}) \\
& +i \widetilde{i d} U(x, \theta, \bar{\theta}) U^{\dagger}(x, \theta, \bar{\theta}),
\end{aligned}
$$

where $\widetilde{A}_{(h)}^{(1)}=d x^{\mu} \mathscr{B}_{\mu}^{(h)}(x, \theta, \bar{\theta})+d \theta \bar{F}^{(h)}(x, \theta, \bar{\theta})+d \bar{\theta} F^{(h)}(x, \theta$, $\bar{\theta})$. In this expression, the superfield $\mathscr{B}_{\mu}^{(h)}(x, \theta, \bar{\theta})$ yields the (anti-)BRST symmetry transformations for the gauge field $A_{\mu}(x)$ and the superfields $\left(F^{(h)}(x, \theta, \bar{\theta}), \bar{F}^{(h)}(x, \theta, \bar{\theta})\right)$ yield the (anti-)BRST symmetry transformations for the ghost and antighost fields, respectively. Here the superscript $(h)$ denotes the expressions of the superfields after the application of the HC. Equation (58) provides an alternative to the $\mathrm{HC}$ in terms of the SUSP unitary operator $U$ and its Hermitian conjugate $U^{\dagger}$ (see, e.g., [18] for details). We shall see below that we can derive the proper (anti-)dual-BRST symmetry transformations for the relevant fields/variables from the equations like (56), (57), and (58) which would be obtained after the application of the duality transformations $\left(A_{\mu} \rightarrow\right.$ $\left.-\varepsilon_{\mu \nu} A^{\nu}, C \rightarrow \bar{C}, \bar{C} \rightarrow C\right)$.

We focus, first of all, on the derivation of the (anti-)coBRST symmetry transformations for the $1 \mathrm{D}$ rigid rotor where the form of the unitary operator and its Hermitian conjugate is the same as given in (56) and (57) with the replacement $x \rightarrow t$ (i.e., $\left.\left.U(x, \theta, \bar{\theta})\right|_{x=t}=U(t, \theta, \bar{\theta})\right)$, where all the fields are functions of $t$ only (i.e., $B(t), C(t), \bar{C}(t))$. There is a duality in the theory, where $\lambda \rightarrow p_{r}, C \rightarrow \bar{C}$, and $\bar{C} \rightarrow C$ for the presence of the (anti-)dual-BRST symmetry transformations $s_{(a) d}$. This is due to the fact that the role of $\lambda, p_{r}, C$, and $\bar{C}$ changes in a symmetrical fashion when we go from the (anti)BRST symmetries to the (anti-)dual-BRST symmetries. A careful and close look at (2) shows that the role of $B$ in the (anti-)BRST symmetry transformations is traded with $(r-a)$ in the (anti-)co-BRST symmetries. Thus, we have the SUSP dual unitary operator and its Hermitian conjugate operator from unitary operators (56) (with the replacement $B \rightarrow(r-$ a)) as

$$
\begin{gathered}
U(t, \theta, \bar{\theta}) \longrightarrow \\
\widetilde{U}(t, \theta, \bar{\theta})=1+\theta(-i C)+\bar{\theta}(-i \bar{C})+\theta \bar{\theta}[(r-a)-\bar{C} C], \\
U^{\dagger}(t, \theta, \bar{\theta}) \longrightarrow \\
\widetilde{U}^{\dagger}(t, \theta, \bar{\theta})=1+\theta(i C)+\bar{\theta}(i \bar{C})+\theta \bar{\theta}[-(r-a)-\bar{C} C],
\end{gathered}
$$

which also satisfy $\widetilde{U} \widetilde{U}^{\dagger}=\widetilde{U}^{\dagger} \widetilde{U}=1$ and they can be exponentiated as

$$
\begin{aligned}
\widetilde{U}(t, \theta, \bar{\theta}) & =\exp [\theta(-i C)+\bar{\theta}(-i \bar{C})+\theta \bar{\theta}(r-a)], \\
\widetilde{U}^{\dagger}(t, \theta, \bar{\theta}) & =\exp [\theta(i C)+\bar{\theta}(i \bar{C})+\theta \bar{\theta}\{-(r-a)\}],
\end{aligned}
$$

so that we have the validity of unitary condition $\widetilde{U} \widetilde{U}^{\dagger}=$ $\widetilde{U}^{\dagger} \widetilde{U}=1$ in a straightforward manner. Now the DHC can be expressed in the following fashion:

$$
\begin{aligned}
p_{r}^{(1)}(t) \longrightarrow & \widetilde{P}_{r}^{(1)(d)}(t, \theta, \bar{\theta}) \\
= & \widetilde{U}(t, \theta, \bar{\theta}) p_{r}^{(1)}(t) \widetilde{U}^{\dagger}(t, \theta, \bar{\theta}) \\
& +i(\widetilde{d} \widetilde{U}(t, \theta, \bar{\theta})) \widetilde{U}^{\dagger}(t, \theta, \bar{\theta}),
\end{aligned}
$$


where $\widetilde{P}_{r}^{(1)(d)}(t, \theta, \bar{\theta})=d t P_{r}^{(d)}(t, \theta, \bar{\theta})+d \theta F^{(d)}(t, \theta, \bar{\theta})+$ $d \bar{\theta} \bar{F}^{(d)}(t, \theta, \bar{\theta})$. It should be noted that we have already taken into account the dual transformations

$$
\begin{gathered}
F^{(d)}(t, \theta, \bar{\theta}) \longrightarrow \bar{F}^{(d)}(t, \theta, \bar{\theta}), \\
\bar{F}^{(d)}(t, \theta, \bar{\theta}) \longrightarrow F^{(d)}(t, \theta, \bar{\theta}),
\end{gathered}
$$

in the definition of the l.h.s. of (61) which yields the expressions of the superfields $\left(P_{r}^{(d)}, F^{(d)}, \bar{F}^{(d)}\right)$ after the application of the DHC. In this connection, it is to be pointed out that the explicit expressions of $P_{r}^{(d)}, F^{(d)}$, and $\bar{F}^{(d)}$ have been already given in (20) and we have the expression for the superexterior derivative as $\widetilde{d}=d t \partial_{t}+d \theta \partial_{\theta}+d \bar{\theta} \partial_{\bar{\theta}}$. Written in the explicit forms, the quantum dual gauge [i.e., (anti-)coBRST] transformation (61) implies the following expressions for $P_{(r)}^{(d)}(t, \theta, \bar{\theta}), F^{(d)}(t, \theta, \bar{\theta})$, and $\bar{F}^{(d)}(t, \theta, \bar{\theta})$ in terms of the SUSP dual unitary operator and its Hermitian conjugate; namely,

$$
\begin{aligned}
& P_{r}^{(d)}(t, \theta, \bar{\theta})=p_{r}(t)+i\left(\partial_{t} \widetilde{U}\right) \widetilde{U}^{\dagger}, \\
& F^{(d)}(t, \theta, \bar{\theta})=i\left(\partial_{\theta} \widetilde{U}\right) \widetilde{U}^{\dagger}, \\
& \bar{F}^{(d)}(t, \theta, \bar{\theta})=i\left(\partial_{\bar{\theta}} \widetilde{U}\right) \widetilde{U}^{\dagger} .
\end{aligned}
$$

The explicit substitution of $\widetilde{U}$ and $\widetilde{U}^{\dagger}$ from (59) into the above relationships yields exactly the same result as (20) for the expansions of $P_{r}^{(d)}, F^{(d)}$, and $\bar{F}^{(d)}$.

In the above, we have constructed a 1-form $p_{(r)}^{(1)}=d t p_{(r)}(t)$ on the $1 \mathrm{D}$ manifold for the derivation of the (anti-)dualBRST symmetries. This should be contrasted with the 1 -form $\lambda^{(1)}=d t \lambda(t)$ that was taken into account in the context of the derivation of the (anti-)BRST symmetries [18]. We have done it because of the fact that there is a duality (i.e., $\lambda \rightarrow p_{r}, C \rightarrow$ $\bar{C}, \bar{C} \rightarrow C)$ in the theory when we go from $s_{(a) b} \rightarrow s_{(a) d}$. Thus, the super 1-form $\tilde{\lambda}^{(1)(h)}(t, \theta, \bar{\theta}) \rightarrow \widetilde{P}_{r}^{(d)}(t, \theta, \bar{\theta})$ such that an appropriate super 1-form $\widetilde{P}_{r}^{(1)}(t, \theta, \bar{\theta})$ is defined, for the derivation of the (anti-)co-BRST symmetry transformations. Thus, now we have $\widetilde{P}_{r}^{(1)}(t, \theta, \bar{\theta})=d t P_{r}(t, \theta, \bar{\theta})+d \theta F(t, \theta, \bar{\theta})+$ $d \bar{\theta} \bar{F}(t, \theta, \bar{\theta})$. We note that the above 1 -form is derived from the definition of super 1-form $\tilde{\lambda}^{(1)}(t, \theta, \bar{\theta})=d t \Lambda(t, \theta, \bar{\theta})+$ $d \theta \bar{F}(t, \theta, \bar{\theta})+d \bar{\theta} F(t, \theta, \bar{\theta})$ that has been used for the derivation of the (anti-)BRST symmetries [18]. From relationship (61), it can be checked that $\widetilde{d} \widetilde{P}_{r}^{(1)(d)}=d p_{r}(t)=0$ (where we have operated by $\widetilde{d}$ from the left on $\widetilde{P}_{r}^{(1)(d)}$ and taken into account the fact that $\tilde{d} p_{r}^{(1)}=d p_{r}^{(1)}=0$ and $\tilde{d} \widetilde{U} \wedge \widetilde{d} \widetilde{U}^{\dagger}=0$ ). To be more precise, it can be checked that $\tilde{d} p_{r}^{(1)}=d p_{r}^{(1)}$ because $p_{r}^{(1)}=d t p_{r}(t)$ and $\partial_{\theta} p_{r}(t)=\partial_{\bar{\theta}} p_{r}(t)=0$. The explicit forms of $\widetilde{d} \widetilde{U}$ and $\widetilde{d} \widetilde{U}^{\dagger}$ are as follows:

$$
\begin{aligned}
& \widetilde{d} \widetilde{U}=d t[\theta(-i \dot{C})+\bar{\theta}(-i \dot{\bar{C}}) \\
&\left.+\theta \bar{\theta}\left\{\frac{d}{d t}(r-a)-\dot{\bar{C}} C-\bar{C} \dot{C}\right\}\right]+d \theta[-i C \\
&+\bar{\theta}\{(r-a)-\bar{C} C\}]+d \bar{\theta}[-i \bar{C} \\
&-\theta\{(r-a)-\bar{C} C\}], \\
& \widetilde{d} \widetilde{U}^{\dagger}=d t[\theta(i \dot{C})+\bar{\theta}(i \dot{\bar{C}}) \\
&\left.+\theta \bar{\theta}\left\{-\frac{d}{d t}(r-a)-\dot{\bar{C}} C-\bar{C} \dot{C}\right\}\right]+d \theta[i C \\
&+\bar{\theta}\{-(r-a)-\bar{C} C\}]+d \bar{\theta}[i \bar{C} \\
&-\theta\{-(r-a)-\bar{C} C\}] .
\end{aligned}
$$

The claim $\widetilde{d} \widetilde{U} \wedge \widetilde{d} \widetilde{U}^{\dagger}=0$ can be proven by collecting all the coefficients of $(d t \wedge d \theta),(d t \wedge d \bar{\theta}),(d \theta \wedge d \theta),(d \bar{\theta} \wedge d \bar{\theta})$, and $(d \theta \wedge d \bar{\theta})$ and showing that these are exactly zero. There is a simpler method to prove this statement by looking carefully at the exponential forms of $U$ and $U^{\dagger}$ [cf. (60)]. We note that the exponents are the same, modulo a sign factor. Therefore, the quantity $\widetilde{d} \widetilde{U} \wedge \tilde{d} \widetilde{U}^{\dagger}$ would imply the wedge product between the same quantities (i.e., exponents). Since the exponents are bosonic in nature, their wedge product would always be zero. Thus, we conclude that $\widetilde{d} \widetilde{U} \wedge \widetilde{d} \widetilde{U}^{\dagger}=0$ which implies that a 2form $\left(\tilde{d} P_{r}^{(d)}=d p_{r}^{(1)}=0\right)$ cannot be defined on a 1D manifold. Hence, the r.h.s. of $\widetilde{d} \widetilde{U} \wedge \widetilde{d} \widetilde{U}^{\dagger}$ is zero.

We have to express the superexpansion of $\Lambda^{(d)}(t, \theta, \bar{\theta})$ in terms of $U$ and $U^{\dagger}$. In this connection, we observe that (anti)dual-BRST invariant quantity of interest is

$$
s_{(a) d}\left[p_{r}-\dot{\lambda}\right]=0 .
$$

According to AVSF, we have the following equality (with $\lambda(t) \rightarrow \Lambda(t, \theta, \bar{\theta})):$

$$
\widetilde{P}_{r}^{(1)(d)}(t, \theta, \bar{\theta})-\tilde{d} \Lambda(t, \theta, \bar{\theta})=p_{r}^{(1)}(t)-d \lambda(t),
$$

where the other symbols have been explained earlier. Taking the input from (61), we obtain the following:

$$
p_{r}(t)+i(\widetilde{d} \widetilde{U}) \widetilde{U}^{\dagger}-\widetilde{d} \Lambda(t, \theta, \bar{\theta})=p_{r}^{(1)}(t)-\dot{\lambda}(t) .
$$

From the above relationship, it is very much evident that we obtain the following relations:

$$
\begin{aligned}
\dot{\Lambda} & =\dot{\lambda}+i\left(\partial_{t} \widetilde{U}\right) \widetilde{U}^{\dagger}, \\
\partial_{\theta} \Lambda & =i\left(\partial_{\theta} \widetilde{U}\right) \widetilde{U}^{\dagger} \\
\partial_{\bar{\theta}} \Lambda & =i\left(\partial_{\bar{\theta}} \widetilde{U}\right) \widetilde{U}^{\dagger}
\end{aligned}
$$


It is very interesting to observe, from (63), that we have the relationships $\partial_{\theta} \Lambda(t, \theta, \bar{\theta})=F^{(d)}(t, \theta, \bar{\theta})$ and $\partial_{\bar{\theta}} \Lambda(t, \theta, \bar{\theta})=$ $\bar{F}^{(d)}(t, \theta, \bar{\theta})$. Thus, taking into account the expressions given in (63) and (67), we have derived the expansion $\Lambda^{(d)}(t, \theta, \bar{\theta})$ [cf. (20)] in terms of the SUSP dual unitary operator and its Hermitian conjugate. Ultimately, we note that the (anti-)co-BRST symmetry invariance of the quantities (i.e., $\left.s_{(a)} d\left[r, \theta, p_{\theta}\right]=0\right)$ can be translated into the generalizations $r(t) \rightarrow R(t, \theta, \bar{\theta})=r(t), \theta(t) \rightarrow \Theta(t, \theta, \bar{\theta})=\theta(t)$ and $p_{\theta}(t) \rightarrow P_{\theta}(t, \theta, \bar{\theta})=p_{\theta}(t)$ (which are primarily trivial generalizations).

We concentrate now on the modified version of 2D Proca theory as well as the anomalous gauge theory and express the DHC and DGIRs in terms of the SUSP dual unitary operator and its Hermitian conjugate. In these theories, there is a duality in the sense that the transformations $A_{\mu} \rightarrow$ $A_{\mu}^{(d)}=-\varepsilon_{\mu \nu} A^{\nu}, C \rightarrow \bar{C}$, and $\bar{C} \rightarrow C$ yield the (anti)dual-BRST symmetry transformations $s_{(a) d}$ from the (anti)BRST symmetry transformations $s_{(a) b}$ for the gauge and (anti-)ghost fields [18]. Thus, we define the dual super 1-form connection, as an input for the derivation of the (anti-)coBRST symmetries, as

$$
\begin{aligned}
\widetilde{A}_{(d)}^{(1)}(x, \theta, \bar{\theta})= & d x^{\mu}\left[-\varepsilon_{\mu \nu} \mathscr{B}_{(d)}^{\nu}(x, \theta, \bar{\theta})\right] \\
& +d \theta F^{(d)}(x, \theta, \bar{\theta})+d \bar{\theta} \bar{F}^{(d)}(x, \theta, \bar{\theta}) .
\end{aligned}
$$

It is to be noted that we have derived $\widetilde{A}_{(d)}^{(1)}(x, \theta, \bar{\theta})$ from the usual super 1-form $\widetilde{A}^{(1)}=d x^{\mu} \mathscr{B}_{\mu}(x, \theta, \bar{\theta})+d \theta \bar{F}(x, \theta, \bar{\theta})+$ $d \bar{\theta} F(x, \theta, \bar{\theta})$ by the replacements $\mathscr{B}_{\mu} \rightarrow-\varepsilon_{\mu \nu} \mathscr{B}^{\nu}, F \rightarrow \bar{F}$, and $\bar{F} \rightarrow F$ due to the presence of duality in our theory. In the context of (anti-)co-BRST symmetries, it will be noted that the usual definition of the super 1-form (i.e., $\widetilde{A}^{(1)}$ ) is taken into account in a subtle manner. Under the (anti-)dualBRST symmetry transformations, the above super 1-form transforms in the superspace as

$$
\begin{aligned}
\widetilde{A}_{(d)}^{(1)}(x, \theta, \bar{\theta})= & \widetilde{U}(x, \theta, \bar{\theta}) A_{(d)}^{(1)}(x) \widetilde{U}^{\dagger}(x, \theta, \bar{\theta}) \\
& +i(\widetilde{d} \widetilde{U}) \widetilde{U}^{\dagger}
\end{aligned}
$$

where $A_{(d)}^{(1)}(x)=d x^{\mu} A_{\mu}^{(d)}$ and, for the modified version of $2 \mathrm{D}$ Proca theory, the forms of SUSP dual unitary operator $\widetilde{U}$ and its Hermitian conjugate $\widetilde{U}^{\dagger}$ are

$$
\begin{aligned}
& \widetilde{U}(x, \theta, \bar{\theta}) \\
& \quad=\exp [\theta(-i \bar{C})+\bar{\theta}(-i C)+\theta \bar{\theta}\{-(E-m \widetilde{\phi})\}], \\
& U^{\dagger}(x, \theta, \bar{\theta})=\exp [\theta(i \bar{C})+\bar{\theta}(i C)+\theta \bar{\theta}(E-m \widetilde{\phi})] .
\end{aligned}
$$

The above can be explicitly written (in terms of coefficients of $\theta, \bar{\theta}$, and $\theta \bar{\theta})$ as

$$
\begin{aligned}
\widetilde{U}(x, \theta, \bar{\theta})= & 1+\theta(-i \bar{C})+\bar{\theta}(-i C) \\
& +\theta \bar{\theta}[-(E-m \widetilde{\phi})-\bar{C} C], \\
\widetilde{U}^{\dagger}(x, \theta, \bar{\theta})= & 1+\theta(i \bar{C})+\bar{\theta}(i C) \\
& +\theta \bar{\theta}[(E-m \tilde{\phi})-\bar{C} C] .
\end{aligned}
$$

The substitution of (72) into (70) yields the following:

$$
\begin{aligned}
\bar{F}^{(d)} & =i\left(\partial_{\bar{\theta}} \widetilde{U}\right) \widetilde{U}^{\dagger}, \\
F^{(d)} & =i\left(\partial_{\theta} \widetilde{U}\right) \widetilde{U}^{\dagger}, \\
\varepsilon_{\mu \nu} \mathscr{B}^{\nu}(x, \theta, \bar{\theta}) & =\varepsilon_{\mu \nu} A^{\nu}(x)-i\left(\partial_{\mu} \widetilde{U}\right) \widetilde{U}^{\dagger},
\end{aligned}
$$

where we have equated the coefficients of $d x^{\mu}, d \theta$, and $d \bar{\theta}$ from the l.h.s. and r.h.s. of (70). The last entry in the above equation leads to the following:

$$
\begin{aligned}
\mathscr{B}_{\mu}^{(d)}= & A_{\mu}(x)-i \varepsilon_{\mu \nu}\left(\partial^{\nu} \widetilde{U}\right) \widetilde{U}^{\dagger} \\
\equiv & A_{\mu}(x)+\theta\left(-\varepsilon_{\mu \nu} \partial^{\nu} C\right)+\bar{\theta}\left(-\varepsilon_{\mu \nu} \partial^{\nu} \bar{C}\right) \\
& +\theta \bar{\theta}\left[i \varepsilon_{\mu \nu}(E-m \widetilde{\phi})\right] \\
\equiv & A_{\mu}(x)+\theta\left(s_{a d} A_{\mu}\right)+\bar{\theta}\left(s_{d} A_{\mu}\right) \\
& +\theta \bar{\theta}\left(s_{d} s_{a d} A_{\mu}\right) .
\end{aligned}
$$

Similarly, we have the following superexpansions in an explicit form:

$$
\begin{aligned}
F^{(d)} & =i\left(\partial_{\theta} \widetilde{U}\right) \widetilde{U}^{\dagger}=C(x)+\bar{\theta}(-[E-m \widetilde{\phi}]) \\
& \equiv C(x)+\bar{\theta}\left(s_{d} C\right), \\
\bar{F}^{(d)} & =i\left(\partial_{\bar{\theta}} \widetilde{U}\right) \widetilde{U}^{\dagger}=\bar{C}(x)+\theta([E-m \widetilde{\phi}]) \\
& \equiv \bar{C}(x)+\theta\left(s_{a d} \bar{C}\right) .
\end{aligned}
$$

Thus, we have derived the proper (anti-)co-BRST symmetry transformations for the basic fields $A_{\mu}(x) C(x)$ and $\bar{C}(x)$ which are common for the modified versions of the $2 \mathrm{D}$ Proca as well as anomalous gauge theories. In the latter case, however, we have to replace $(E-m \widetilde{\phi})$ by $\mathscr{B}(x)$ in the definition of the SUSP dual unitary operator and its Hermitian conjugate. We shall now focus on the derivation of proper (anti-)co-BRST symmetries for the additional fields in these theories.

Let us express the superfield $\widetilde{\phi}^{(d)}(x, \theta, \bar{\theta})$ in the language of the SUSP dual unitary operator and its Hermitian conjugate. We have seen that $s_{(a) d}[E-m \widetilde{\phi}]=0$ due to the on-shell nilpotency (i.e., $s_{(a) d}^{2} C=s_{(a) d}^{2} \bar{C}=0$ ) in the 
theory because of the fact that the (anti-)ghost fields $(\bar{C}) C$ obey the on-shell conditions: $\left(\square+m^{2}\right) C=0$ and $(\square+$ $\left.m^{2}\right) \bar{C}=0$. Thus, the combination $[E-m \widetilde{\phi}]$ is an (anti)co-BRST invariant quantity which can be generalized onto the $(2,2)$-dimensional supermanifold due to AVSF. This can be expressed in the language of superfields, derived after the application of the DHCs and DGIRs, as

$$
\begin{gathered}
\varepsilon^{\mu \nu} \partial_{\mu} \mathscr{B}_{\nu}^{(d)}(x, \theta, \bar{\theta})+m \widetilde{\Phi}(x, \theta, \bar{\theta}) \\
=\varepsilon^{\mu \nu} \partial_{\mu} A_{\nu}(x)+m \widetilde{\phi}(x) .
\end{gathered}
$$

Using the expression for $\mathscr{B}_{\mu}^{(d)}(x, \theta, \bar{\theta})$ from (74), we have

$$
\begin{aligned}
& \varepsilon^{\mu \nu} \partial_{\mu}\left[A_{\nu}(x)-i \varepsilon_{\nu \lambda}\left(\partial^{\lambda} \widetilde{U}\right) \widetilde{U}^{\dagger}\right]+m \widetilde{\Phi}(x, \theta, \bar{\theta}) \\
& =\varepsilon^{\mu \nu} \partial_{\mu} A_{\nu}(x)+m \widetilde{\phi}(x) .
\end{aligned}
$$

This relation, finally, leads to the following expression for $\widetilde{\Phi}^{(d)}(x, \theta, \bar{\theta})$ in terms of the SUSP dual unitary operators (i.e., $\widetilde{U}$ and $\left.\widetilde{U}^{\dagger}\right)$; namely,

$$
\begin{aligned}
\widetilde{\Phi}^{(d)}(x, \theta, \bar{\theta})= & \widetilde{\phi}(x)+\frac{i}{m}\left(\partial_{\mu} \widetilde{U}\right)\left(\partial^{\mu} \widetilde{U}^{\dagger}\right) \\
& +\frac{i}{m}(\square \widetilde{U}) \widetilde{U}^{\dagger} .
\end{aligned}
$$

It is very interesting to check that the r.h.s. yields expansions (34) when we use the on-shell conditions: $\left(\square+m^{2}\right) C=0$, $\left(\square+m^{2}\right) \bar{C}=0$, and $\left(\square+m^{2}\right) \mathscr{B}=0$. It is important to point out that the contributions, from the second term of (78), cancel out the extra piece that emerges from the last term on the r.h.s. of (78). Thus, we have expressed all the nontrivial nilpotent and anticommuting (anti-)co-BRST symmetry transformations of the 2D Proca theory in terms of the SUSP dual unitary operators $\widetilde{U}$ and $\widetilde{U}^{\dagger}$.

A close look and careful observations of (34) and (36) demonstrate that the expansions are very similar and they differ only by a factor of $m$. Thus, it is very elementary to note that expansions (36) can be expressed in terms of the SUSP operators (i.e., SUSP unitary operator $\widetilde{U}$ and its Hermitian conjugate $\widetilde{U}^{\dagger}$ ) as follows:

$$
\Phi^{(d)}(x, \theta, \bar{\theta})=\phi(x)+i\left(\partial_{\mu} \widetilde{U}\right)\left(\partial^{\mu} \widetilde{U}^{\dagger}\right)+i(\square \widetilde{U}) \widetilde{U}^{\dagger} .
$$

With the substitution of the expressions for $\widetilde{U}$ and $\widetilde{U}^{\dagger}$ from (71) and (72) (with the replacement $(E-m \widetilde{\phi}) \rightarrow \mathscr{B}$ ), we obtain the r.h.s. of expansion (36) from the r.h.s. of the above relationship. We concentrate now on the alternative to the expansion $\Sigma^{(d)}(x, \theta, \bar{\theta})$ (cf. (40)) in the language of the SUSP unitary operator $\widetilde{U}$ and its Hermitian conjugate $\widetilde{U}^{\dagger}$. This can be derived from the restrictions (due to $s_{(a) d}[(a-1) \sigma(x)-$ $\phi(x)=0]$ ) on the superfields, due to the basic tenets of AVSF, as

$$
\begin{aligned}
& (a-1) \Sigma(x, \theta, \bar{\theta})-\Phi^{(d)}(x, \theta, \bar{\theta}) \\
& \quad=(a-1) \sigma(x)-\phi(x) .
\end{aligned}
$$

The substitution of (79) into the above equation yields the following:

$$
\begin{aligned}
\Sigma^{(d)}(x, \theta, \bar{\theta})= & \sigma(x)+\frac{i}{(a-1)}\left(\partial_{\mu} \widetilde{U}\right)\left(\partial^{\mu} \widetilde{U}^{\dagger}\right) \\
& +\frac{i}{(a-1)}(\square \widetilde{U}) \widetilde{U}^{\dagger} .
\end{aligned}
$$

Thus, we have obtained all the nontrivial (anti-)co-BRST symmetry transformations for the modified version of $2 \mathrm{D}$ anomalous gauge theory in the terminology of SUSP dual unitary operator $\widetilde{U}$ and its Hermitian conjugate $\widetilde{U}^{\dagger}$. In other words, we conclude that the precise derivations of $\widetilde{U}$ and $\widetilde{U}^{\dagger}$ provide the alternatives to the DHCs and DGIRs that are exploited within the framework of AVSF for the derivation of the (anti-)co-BRST symmetry transformations. Thus, the precise forms of $\widetilde{U}$ and $\widetilde{U}^{\dagger}$ are physically important.

Finally, we focus on the alternative to the DHC and DGIRs in the context of 2D self-dual bosonic field theory. Here the SUSP dual unitary operators $\widetilde{U}$ and $\widetilde{U}^{\dagger}$ would be exactly the same as in (59) with some replacements in view of the (anti-)co-BRST symmetry transformations (2) vis-à-vis (9). Thus, we have now

$$
\begin{gathered}
\widetilde{U}(x, \theta, \bar{\theta})=\exp [\theta(-i \bar{C})+\bar{\theta}(-i C) \\
\left.+\theta \bar{\theta}\left\{-\frac{1}{2}\left(\dot{\phi}-\dot{v}+v^{\prime}-\phi^{\prime}\right)\right\}\right], \\
\widetilde{U}^{\dagger}(x, \theta, \bar{\theta})=\exp [\theta(i \bar{C})+\bar{\theta}(i C) \\
\left.+\theta \bar{\theta}\left\{\frac{1}{2}\left(\dot{\phi}-\dot{v}+v^{\prime}-\phi^{\prime}\right)\right\}\right] .
\end{gathered}
$$

We define the dual super 1-form as follows:

$$
\begin{aligned}
\Phi^{(1)}(x, \theta, \bar{\theta})= & d t 2 \Phi^{(d)}(x, \theta, \bar{\theta})+d \theta F^{(d)}(x, \theta, \bar{\theta}) \\
& +d \bar{\theta} \bar{F}^{(d)}(x, \theta, \bar{\theta}),
\end{aligned}
$$

where the expansions for $\Phi^{(d)}(x, \theta, \bar{\theta}), F^{(d)}(x, \theta, \bar{\theta})$, and $\bar{F}^{(d)}(x, \theta, \bar{\theta})$ have to be determined in terms of $\widetilde{U}$ and $\widetilde{U}^{\dagger}$ listed in (82). We point out that a factor of two has been taken into account in (83) because of the observation that $\left(s_{d} \phi=\dot{\bar{C}} / 2, s_{a d} \phi=\dot{C} / 2\right)$ [cf. (9)]. The transformations of the super 1-form (83) in the superspace are

$$
\Phi^{(1)(d)}(x, \theta, \bar{\theta})=\widetilde{U} \phi^{(1)}(x) \widetilde{U}^{\dagger}+i(\widetilde{d} \widetilde{U}) \widetilde{U}^{\dagger},
$$

where $\phi^{(1)}(x)=d t[2 \phi(x)]$ is a 1 -form on the 1D submanifold of the general $2 \mathrm{D}$ ordinary spacetime manifold. The above equation, taking into account definition (83), is as follows in the component form:

$$
\begin{aligned}
2 \Phi^{(d)}(x, \theta, \bar{\theta}) & =2 \phi(x)+i\left(\partial_{t} \widetilde{U}\right) \widetilde{U}^{\dagger}, \\
F^{(d)}(x, \theta, \bar{\theta}) & =i\left(\partial_{\theta} \widetilde{U}\right) \widetilde{U}^{\dagger}, \\
\bar{F}^{(d)}(x, \theta, \bar{\theta}) & =i\left(\partial_{\bar{\theta}} \widetilde{U}\right) \widetilde{U}^{\dagger},
\end{aligned}
$$


where we have taken into account the comparison of the coefficients of $d x^{\mu}, d \theta$, and $d \bar{\theta}$ from r.h.s. and 1.h.s. of (84) (with input from (83)). The substitution of the explicit form of $\widetilde{U}$ and $\widetilde{U}^{\dagger}$ from (82) leads to the following expansions from the superfields (cf. (55)):

$$
\begin{aligned}
& \Phi^{(d)}(x, \theta, \bar{\theta})= \phi(x)+\theta\left(\frac{\dot{C}}{2}\right)+\bar{\theta}\left(\frac{\dot{\bar{C}}}{2}\right) \\
&+\theta \bar{\theta}\left(-\frac{i}{4} \frac{\partial}{\partial t}\left[\dot{\phi}-\dot{v}+v^{\prime}-\phi^{\prime}\right]\right), \\
& F^{(d)}(x, \theta, \bar{\theta})= C(x)+\bar{\theta}\left(-\frac{i}{2}\left[\dot{\phi}-\dot{v}+v^{\prime}-\phi^{\prime}\right]\right), \\
& \bar{F}^{(d)}(x, \theta, \bar{\theta})=\bar{C}(x)+\theta\left(\frac{i}{2}\left[\dot{\phi}-\dot{v}+v^{\prime}-\phi^{\prime}\right]\right) .
\end{aligned}
$$

A close look at the expansions shows that we have already derived the (anti-)co-BRST symmetry transformations $s_{(a) d}$ for the fields $\phi(x), C(x)$, and $\bar{C}(x)$. We note that $s_{(a) d}[\phi-v]=$ 0 . This observation implies immediately, due to the basic tenets of AVSF, that we have the following expansion of the superfield corresponding to the WZ-field $v(x)$; namely,

$$
\begin{aligned}
v(x) \longrightarrow & V^{(d)}(x, \theta, \bar{\theta}) \\
= & v(x)+\theta\left(\frac{\dot{C}}{2}\right)+\bar{\theta}\left(\frac{\dot{\bar{C}}}{2}\right) \\
& +\theta \bar{\theta}\left(-\frac{i}{4} \frac{\partial}{\partial t}\left[\dot{\phi}-\dot{v}+v^{\prime}-\phi^{\prime}\right]\right) \\
\equiv & v(x)+\theta\left(s_{a d} v(x)\right)+\bar{\theta}\left(s_{d} v(x)\right) \\
& +\theta \bar{\theta}\left(s_{d} s_{a d} v(x)\right),
\end{aligned}
$$

where the (anti-)co-BRST symmetry transformations $s_{(a) d}$ are listed in (9). Equation (87) can also be written in terms of $\widetilde{U}$ and $\widetilde{U}^{\dagger}$ as

$$
2 V^{(d)}(x, \theta, \bar{\theta})=2 v(x)+i\left(\partial_{t} \widetilde{U}\right) \widetilde{U}^{\dagger} .
$$

This is due to the fact that a super 1-form can be written exactly like (83) in terms of $V^{(d)}(x, \theta, \bar{\theta}), F^{(d)}(x, \theta, \bar{\theta})$, and $\bar{F}^{(d)}(x, \theta, \bar{\theta})$. It goes without saying that we can repeat the above exercise to obtain the superspace transformation like (85) and (86).

We observe that $s_{(a) d}[\dot{\lambda}-2 \phi]=s_{(a) d}[\dot{\lambda}-2 v]=0$. Thus, we have the following restrictions (due to these invariances) on the superfields, defined on (2,2)-dimensional supermanifold, according to basic tenets of AVSF; namely,

$$
\begin{aligned}
& \dot{\Lambda}(x, \theta, \bar{\theta})-2 \Phi^{(d)}(x, \theta, \bar{\theta})=\dot{\lambda}(x)-2 \phi(x), \\
& \dot{\Lambda}(x, \theta, \bar{\theta})-2 V^{(d)}(x, \theta, \bar{\theta})=\dot{\lambda}(x)-2 v(x),
\end{aligned}
$$

which implies that the superfield $\Lambda^{(d)}(x, \theta, \bar{\theta})$ can be expressed (from both the above relationships) in terms of the SUSP dual unitary operators $\widetilde{U}$ and $\widetilde{U}^{\dagger}$ as

$$
\dot{\Lambda}^{(d)}(x, \theta, \bar{\theta})=\dot{\lambda}(x)+i\left(\partial_{t} \widetilde{U}\right) \widetilde{U}^{\dagger},
$$

if we use the appropriate relationships quoted in (85) and (88). The above expression finally leads to the following expansions along $(\theta, \bar{\theta})$ directions:

$$
\begin{aligned}
\Lambda^{(d)}(x, \theta, \bar{\theta})= & \lambda(x)+\theta(C)+\bar{\theta}(\bar{C}) \\
& +\theta \bar{\theta}\left(-\frac{i}{2}\left[\dot{\phi}-\dot{v}+v^{\prime}-\phi^{\prime}\right]\right) \\
\equiv & \lambda(x)+\theta\left(s_{a d} \lambda(x)\right)+\bar{\theta}\left(s_{d} \lambda(x)\right) \\
& +\theta \bar{\theta}\left(s_{d} s_{a d} \lambda(x)\right) .
\end{aligned}
$$

Thus, we have provided the alternatives to the DHC and DGIRs used, in Section 3, in the language of $\widetilde{U}$ and $\widetilde{U}^{\dagger}$ and obtained all the nontrivial (anti-)co-BRST symmetries of the $2 \mathrm{D}$ self-dual bosonic field theory. We conclude this section with the remarks that SUSP dual unitary operators $\widetilde{U}$ and $\widetilde{U}^{\dagger}$ provide the alternatives to the DHC and DGIRs within the framework of AVSF where the explicit group structure is maintained.

\section{Conclusions}

For the Abelian 1-form $U(1)$ gauge theories, it is important to have explicit existence and appearance of the group structure in any kind of computation. The SUSP dual unitary operator and its Hermitian conjugate do exactly the same job in our present endeavor and that is why their derivation is important. In our earlier works [19, 22], we have explicitly derived the exact form of the SUSP unitary operator and its Hermitian conjugate for the cases of the interacting (i) 4D Abelian $U(1)$ gauge theory with the Dirac and complex scalar fields and (ii) $4 \mathrm{D}$ non-Abelian $\mathrm{SU}(N)$ gauge theory with Dirac fields, in the context of nilpotent (anti-)BRST symmetries. The universal nature of the SUSP unitary operator and its Hermitian conjugate has also been established in our recently published work [18] for the case of the $1 \mathrm{D}$ and 2D Abelian $U(1)$ gauge theories. In fact, we have been able to derive the dual unitary operator and its Hermitian conjugate from the above universal unitary operator by exploiting the virtues of the duality symmetry in our theory where $C \rightarrow \bar{C}, \bar{C} \rightarrow C$, and $A_{\mu} \rightarrow-\varepsilon_{\mu \nu} A^{\nu}$. As it turns out, we observe that the mathematical form of the SUSP dual unitary operator and its Hermitian conjugate is universal in exactly the same way as the SUSP unitary operator and its Hermitian conjugate are (see, e.g., [18]).

We would like to dwell a bit on the duality aspects of our statement. In the case of 2D Abelian 1-form gauge theory, it can be seen that the self-duality condition $* A^{(1)}=$ $*\left(d x^{\mu} A_{\mu}\right)=\varepsilon^{\mu \nu} d x_{\nu} A_{\mu} \equiv d x^{\mu}\left(-\varepsilon_{\mu \nu} A^{\nu}\right) \equiv d x^{\mu} A_{\mu}^{(d)}$, where 
$A_{\mu}^{(d)}=-\varepsilon_{\mu \nu} A^{\nu}$ is the dual 1-form potential corresponding to the Abelian 1-form potential $A_{\mu}$. Furthermore, we observe that when we go from the (anti-)BRST symmetries (particularly in the ghost sector of our theory), there is a transformation from $C \rightarrow \bar{C}$ and $\bar{C} \rightarrow C$. Thus, for a 2D 1-form theory, the transformations $A_{\mu} \rightarrow-\varepsilon_{\mu \nu} A^{v}, C \rightarrow \bar{C}$, and $\bar{C} \rightarrow C$ are the duality transformations which have been exploited in the definition of super 1-forms (cf. (69), (83)). However, in the case of $1 \mathrm{D}$ Abelian 1-form theory (i.e., a rigid rotor), we observe that there is a duality: $\lambda \rightarrow p_{r}$, $C \rightarrow \bar{C}$, and $\bar{C} \rightarrow C$. This observation has been exploited in the statements that have followed (61) and (63) in the definition of $P_{(d)}^{(1)}(x, \theta, \bar{\theta})$. Similar kinds of arguments have been exploited in the case of $2 \mathrm{D}$ self-dual field theory where we have expressed the DHC and DGIRs in the language of $\widetilde{U}$ and $\widetilde{U}^{\dagger}$.

In our present endeavor, we have applied the AVSF to derive the (anti-)co-BRST symmetry transformations for a new model in $2 \mathrm{D}$. This model is nothing but the modified version of the $2 \mathrm{D}$ anomalous gauge theory which has already been proven to provide a tractable model for the Hodge theory [16]. Thus, it is a novel result in our present endeavor. The precise derivation of the (anti-)co-BRST symmetries establishes the sanctity and correctness of the working-rule that has been laid down for the Hodge duality $(\star)$ operation on the $(1,2)$ - and $(2,2)$-dimensional supermanifolds [20]. Thus, we conclude that the AVSF is a powerful theoretical technique that can be applied to interesting physical systems and one can derive the appropriate form of the BRST-type symmetries. The key concepts (which play important roles in the application of the AVSF) are the DHC and DGIRs. One of the key observations of our present endeavor is the fact that the geometrical meaning of the (anti-)co-BRST symmetries, in the language of the translational generators $\left(\partial_{\theta}, \partial_{\bar{\theta}}\right)$ along the Grassmannian directions of the appropriately chosen supermanifold, remains the same when we exploit the theoretical strength of the DHC and DGIRs.

We would like to lay emphasis on the fact that the models of the Abelian 1-form gauge theories in $1 \mathrm{D}$ and 2D (which have been considered in our present endeavor) are interesting because these models provide the tractable physical examples of Hodge theory within the framework of BRST formalism [13-17]. Such models are mathematically as well as physically very rich because there are many continuous symmetries in the theory which enable these theories to be quantized without the definition of the canonical conjugate momenta corresponding to the fields of these theories (see, e.g., [24, $25])$. In the context of gauge theories, it has been shown, in our earlier works $[24,25]$, that there exist six continuous internal symmetries for such theories which are so powerful that they lead to the canonical quantization of these theories at the level of creation and annihilation operators. The above symmetries have also played very important roles in the proof of 2D (non-)Abelian 1-form gauge theories (without any interaction with matter fields) to be a new class [26] of topological field theories (TFTs) that capture a few key aspects of the Witten-type TFTs and some salient features of the Schwartz-type TFTs.
We have succeeded in obtaining universal SUSP unitary operator and its Hermitian conjugate that are primarily connected with the (anti-)BRST symmetries in the cases of 4D interacting Abelian 1-form gauge theories with Dirac fields and $2 \mathrm{D}$ and $1 \mathrm{D}$ Abelian gauge theories. In our present endeavor, we have obtained the SUSP dual unitary operator and its Hermitian conjugate in the cases of 2D and 1D Abelian 1 -form gauge theories that are connected with the (anti)co-BRST symmetry transformations. One of the immediate goals for us is to extend our work to the $2 \mathrm{D}$ non-Abelian 1 -form gauge theory (without any interaction with matter fields) so that we could derive the SUSP unitary operator and its Hermitian conjugate as well as the SUSP dual unitary operator and its Hermitian conjugate. This is essential because we have already shown that this $2 \mathrm{D}$ non-Abelian model is an example of the Hodge theory where the (anti)BRST and (anti-)co-BRST symmetries exist along with other internal symmetries. We have already made some progress in this direction and our results would be reported in our future publication.

\section{Competing Interests}

The authors declare that they have no competing interests.

\section{Acknowledgments}

One of the authors (T. Bhanja) would like to gratefully acknowledge the financial support from CSIR, Govt. of India, New Delhi, under its SRF-scheme. Another author (N. Srinivas) is grateful to the BHU-fellowship for financial support. The present investigation has been carried out under the above financial support.

\section{References}

[1] P. A. M. Dirac, Lectures on Quantum Mechanics, Belfer Graduate School of Science, Yeshiva University Press, New York, NY, USA, 1964.

[2] K. Sundermeyer, Constrained Dynamics, vol. 169 of Lecture Notes in Physics, Springer, Berlin, Germany, 1982.

[3] G. Curci and R. Ferrari, "Slavnov transformations and supersymmetry," Physics Letters B, vol. 63, p. 91, 1976.

[4] J. Thierry-Mieg, "Geometrical reinterpretation of FaddeevPopov ghost particles and BRS transformations," Journal of Mathematical Physics, vol. 21, no. 12, pp. 2834-2838, 1980.

[5] M. Quiros, F. J. De Urries, J. Hoyos, M. L. Mazon, and E. Rodrigues, "Geometrical structure of Faddeev-Popov fields and invariance properties of gauge theories," Journal of Mathematical Physics, vol. 22, no. 8, p. 1767, 1981.

[6] L. Bonora and M. Tonin, "Superfield formulation of extended BRS symmetry," Physics Letters B, vol. 98, no. 1-2, pp. 48-50, 1981.

[7] L. Bonora, P. Pasti, and M. Tonin, "Geometric description of extended BRS symmetry in superfield formulation," Il Nuovo Cimento A, vol. 63, no. 3, pp. 353-364, 1981.

[8] L. Bonora and P. Cotta-Ramusino, "Some remarks on BRS transformations, anomalies and the cohomology of the Lie algebra of the group of gauge transformations," Communications in Mathematical Physics, vol. 87, no. 4, pp. 589-603, 1983. 
[9] R. Delbourgo and P. D. Jarvis, "Extended BRS invariance and osp(4/2) supersymmetry," Journal of Physics A: Mathematical and General, vol. 15, p. 611, 1981.

[10] R. Delbourgo, P. D. Jarvis, and G. Thompson, "Local OSp(4/2) supersymmetry and extended BRS transformations for gravity," Physics Letters B, vol. 109, no. 1-2, pp. 25-27, 1982.

[11] R. P. Malik, "Abelian 2-form gauge theory: superfield formalism," The European Physical Journal C, vol. 60, no. 3, pp. 457470, 2009.

[12] R. P. Malik, "Unique nilpotent symmetry transformations for matter fields in QED: augmented superfield formalism," The European Physical Journal C, vol. 47, no. 1, pp. 227-234, 2006.

[13] S. Gupta and R. P. Malik, "Rigid rotor as a toy model for Hodge theory," The European Physical Journal C, vol. 68, no. 1, pp. 325$335,2010$.

[14] T. Bhanja, D. Shukla, and R. P. Malik, "Novel symmetries in the modified version of two dimensional Proca theory," The European Physical Journal C, vol. 73, article 2535, 2013.

[15] A. Shukla, S. Krishna, and R. P. Malik, "Augmented superfield approach to nilpotent symmetries of the modified version of $2 \mathrm{D}$ Proca theory," Advances in High Energy Physics, vol. 2015, Article ID 258536, 21 pages, 2015.

[16] S. Gupta, R. Kumar, and R. P. Malik, "On free 4D Abelian 2form and anomalous 2D Abelian 1-form gauge theories," The European Physical Journal C, vol. 65, article 311, 2010.

[17] D. Shukla, T. Bhanja, and R. P. Malik, "Self-dual chiral boson: augmented superfield approach," The European Physical Journal C, vol. 74, article 3025, 2014.

[18] T. Bhanja, N. Srinivas, and R. P. Malik, "Universal superspace unitary operator for some interesting Abelian models: superfield approach," Advances in High Energy Physics, vol. 2016, Article ID 3673206, 11 pages, 2016.

[19] T. Bhanja, D. Shukla, and R. P. Malik, "Superspace unitary operator in superfield approach to non-abelian gauge theory with Dirac fields," Advances in High Energy Physics, vol. 2016, Article ID 6367545, 11 pages, 2016.

[20] R. P. Malik, "Hodge duality operation and its physical applications on supermanifolds," International Journal of Modern Physics A, vol. 21, no. 16, pp. 3307-3336, 2006.

[21] D. Shukla, T. Bhanja, and R. P. Malik, "Supervariable approach to the nilpotent symmetries for a toy model of the hodge theory," Advances in High Energy Physics, vol. 2016, Article ID 2618150, 13 pages, 2016.

[22] D. Shukla, T. Bhanja, and R. P. Malik, "Superspace unitary operator in QED with Dirac and complex scalar fields: superfield approach," Europhysics Letters, vol. 112, no. 1, Article ID 11001, 2015.

[23] N. Nakanishi and I. Ojima, Covariant Operator Formalism of Gauge Theory and Quantum Gravity, World Scientific, Singapore, 1990.

[24] R. Kumar, S. Gupta, and R. P. Malik, "Basic brackets of a 2D model for the hodge theory without its canonical conjugate momenta," International Journal of Theoretical Physics, vol. 55, no. 6, pp. 2857-2869, 2016.

[25] N. Srinivas, A. Shukla, and R. P. Malik, " $N=2$ supersymmetric harmonic oscillator: basic brackets without canonical conjugate momenta," International Journal of Modern Physics A, vol. 30, no. 30, Article ID 1550166, 12 pages, 2015.

[26] R. P. Malik, "New topological field theories in two dimensions," Journal of Physics A: Mathematical and General, vol. 34, no. 19, pp. 4167-4181, 2001. 

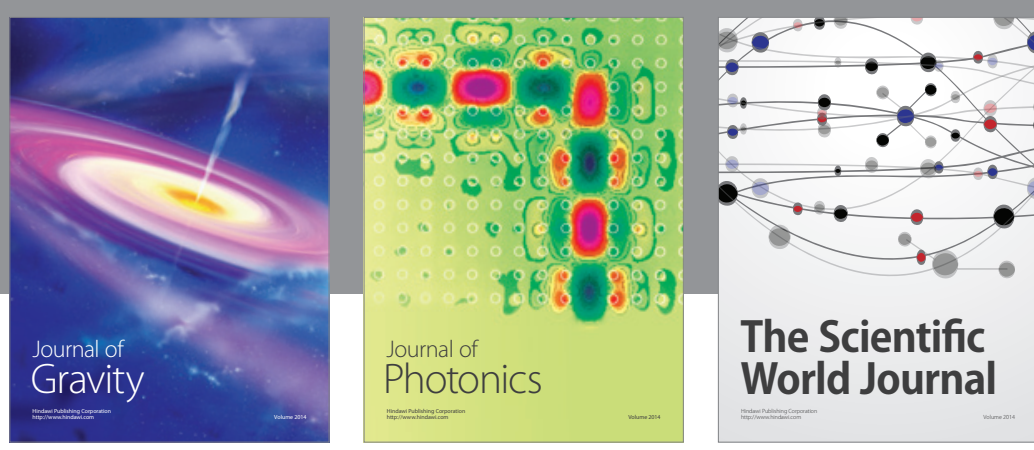

The Scientific World Journal
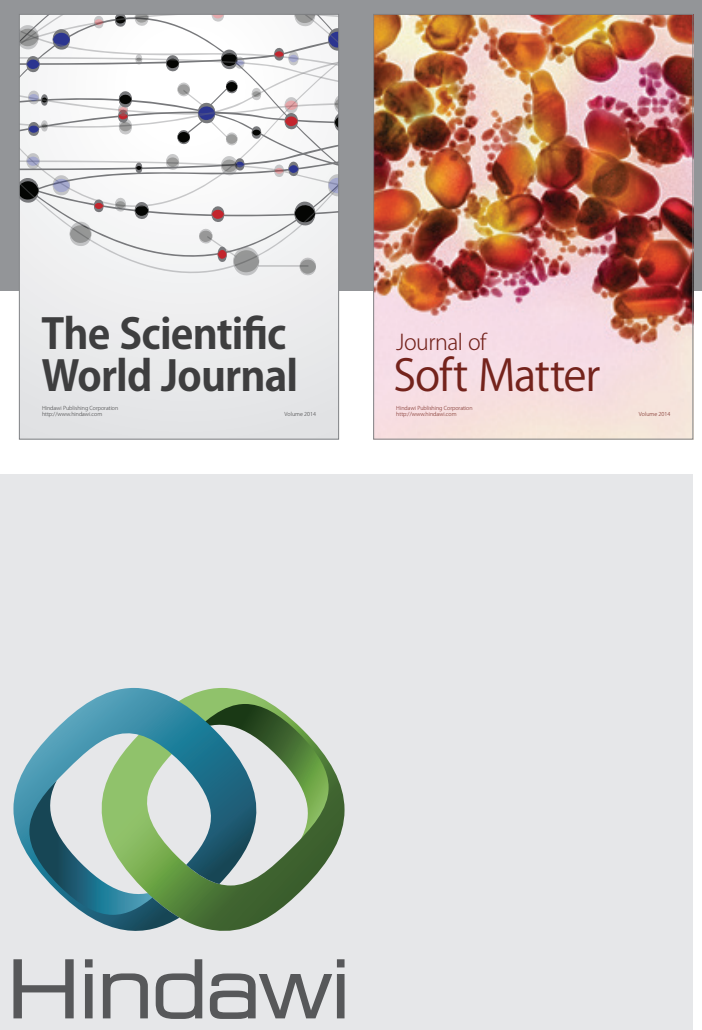

Submit your manuscripts at

http://www.hindawi.com

nternational Journal of

Statistical Mechanics
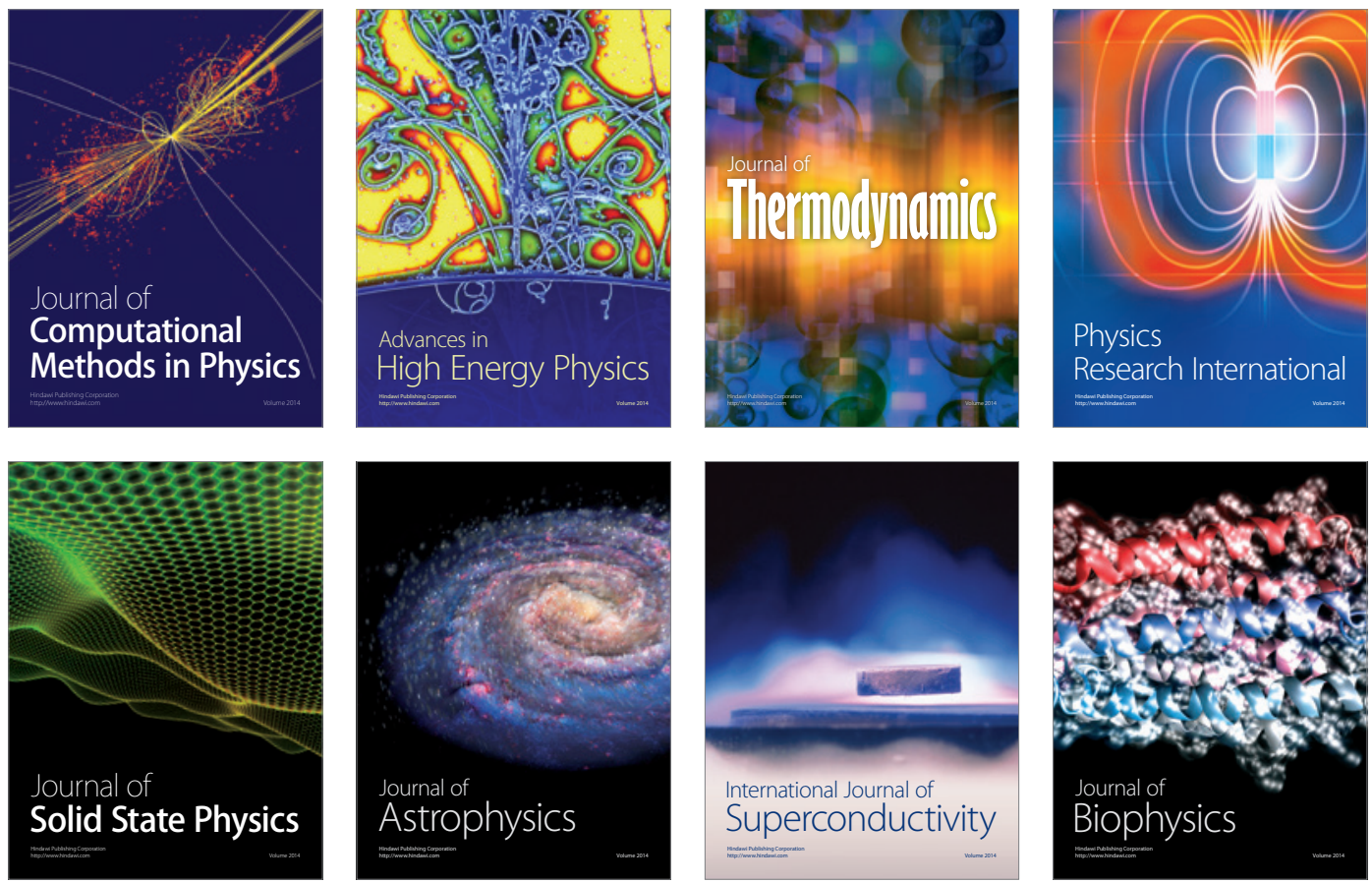
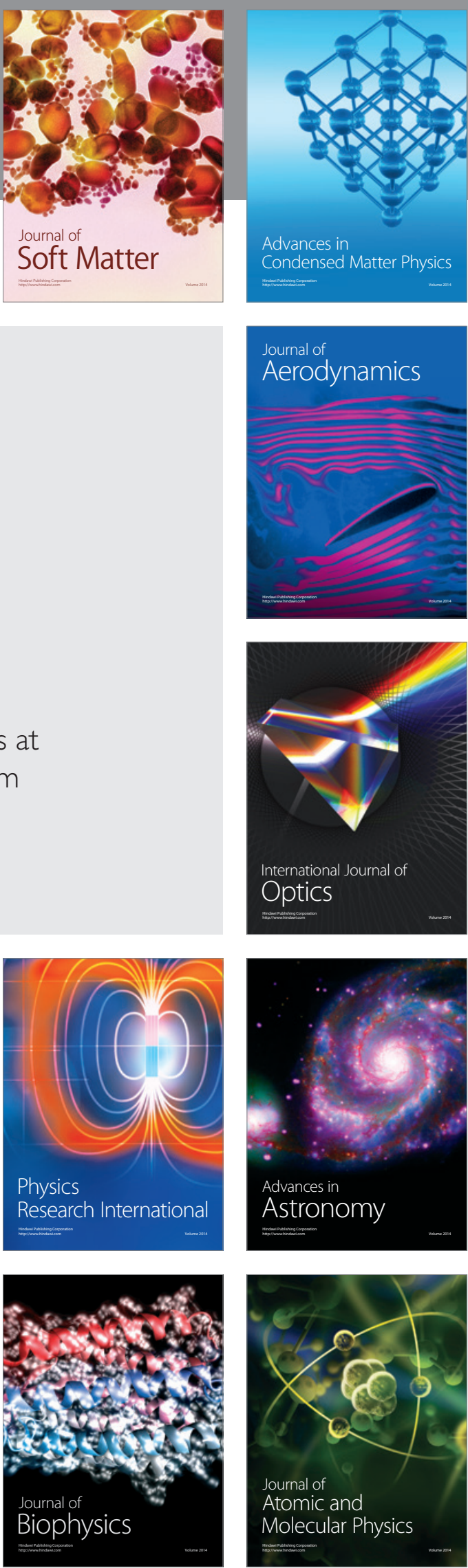